\title{
Mixed hidden sector-visible sector dark matter and observation of a CP odd Higgs boson at HL-LHC and HE-LHC
}

\author{
Amin Aboubrahim* and Pran Nath ${ }^{\dagger}$ \\ Department of Physics, Northeastern University, Boston, Massachusetts 02115-5000, USA
}

(Received 13 May 2019; published 29 July 2019)

\begin{abstract}
It is very likely that, similarly to the case of visible matter, dark matter (DM) is composed of more than one stable component. In this work, we investigate a two-component dark matter with one component from the visible sector and the other from the hidden sector. Specifically, we consider a $U(1)_{X}$ hidden-sector extension of the minimal supersymmetric standard model with supergravity (MSSM/SUGRA), where we allow for kinetic and Stueckelberg mass mixing between the two Abelian $U(1)$ 's, i.e., $U(1)_{X}$ and $U(1)_{Y}$. We further assume that the hidden sector has chiral matter which leads to a Dirac fermion as a candidate for dark matter. The lightest neutralino in the visible sector and the Dirac fermion in the hidden sector then constitute the two components of dark matter. We investigate in particular MSSM/SUGRA models with radiative breaking occurring on the hyperbolic branch where the Higgs mixing parameter $\mu$ is small (the order of the electroweak scale), which leads to the lightest neutralino being dominantly a Higgsino. While dark matter constituted only of Higgsinos is significantly constrained by data on dark matter relic density and by limits on the spin-independent proton-DM scattering cross section, consistency with data can be achieved if only a fraction of the dark matter relic density is constituted of Higgsinos, with the rest coming from the hidden sector. An aspect of the proposed model is the prediction of a relatively light $C P$-odd Higgs $A$ (as well as a $C P$-even $H$ and a charged Higgs $H^{ \pm}$) which is observable in the High-Luminosity and High-Energy Large Hadron Collider projects (HL-LHC and HE-LHC). We perform a detailed collider analysis search for the $C P$-odd Higgs using boosted decision trees in $\tau_{h} \tau_{h}$ final states and compare the discovery potential at the HL-LHC and HE-LHC. We show that while several of the points among our benchmarks may be observable at the HL-LHC, all of them are visible at the HE-LHC with much lower integrated luminosities, thus reducing significantly the run-time for discovery. Thus, the discovery of a $C P$-odd Higgs would lend support to the existence of the hyperbolic branch, a small $\mu$, and point to the multicomponent nature of dark matter. It is also shown that a part of the parameter space of the extended model can be probed in next-generation direct-detection experiments such as XENONnT and LUX-ZEPLIN.
\end{abstract}

DOI: $10.1103 /$ PhysRevD.100.015042

\section{INTRODUCTION}

The discovery of the Higgs boson at the Large Hadron Collider (LHC) at $\sim 125 \mathrm{GeV}[1,2]$ gives strong support for supersymmetry (SUSY). This is so because within the standard model (SM), the Higgs boson mass can lie in a wide range up to several hundred $\mathrm{GeV}$ in mass [3], while within supersymmetry- or supergravity-unified models (for a review, see, e.g., Ref. [4]), the mass of the Higgs boson is predicted to lie below $130 \mathrm{GeV}$ [5-7]. In addition to the fact that LHC data respect the supersymmetric limit, stability of

\footnotetext{
a.abouibrahim@northeastern.edu

p.nath@northeastern.edu
}

Published by the American Physical Society under the terms of the Creative Commons Attribution 4.0 International license. Further distribution of this work must maintain attribution to the author(s) and the published article's title, journal citation, and DOI. Funded by SCOAP ${ }^{3}$. the vacuum can be preserved within supersymmetry up to the Planck scale, while within the standard model the vacuum stability holds only till around $10^{10} \mathrm{GeV}[8,9]$. However, as is well known, the Higgs boson mass at $\sim 125 \mathrm{GeV}$ requires a large loop correction within the minimal supersymmetric standard model with supergravity (MSSM/SUGRA), which in turn implies that the size of weak-scale supersymmetry is large, lying in the several$\mathrm{TeV}$ region. This also explains why SUSY has not been observed at accelerators thus far. The large size of weakscale supersymmetry also has implications for dark matter (DM). In SUGRA-unified models, the low-energy sparticle spectrum determined by running the renormalization group equations (RGEs) and radiative breaking of the electroweak symmetry has several branches. On one branch, the ellipsoidal branch, the Higgs mixing parameter $\mu$ is large, and the lightest supersymmetric particle (LSP), the lightest neutralino, is typically a bino. However, in the early Universe, the binos are not annihilated efficiently, leading 
to an LSP relic density far in excess of the current experiment [10]. Here one way to reduce the relic density is through the utilization of coannihilation. Other possibilities within SUGRA models to get conformity with the relic density constraint include a wino-like dark matter or a Higgsino-like dark matter.

In this work, we focus on SUGRA models on the hyperbolic branch with a small $\mu$ (of the order of the electroweak scale) where the LSP is Higgsino-like. Indeed, within radiative breaking of the electroweak symmetry, Higgsino-like dark matter can arise naturally on the hyperbolic branch when $\mu$ is small [11-13] (for related works, see, e.g., Refs. [14-17]). Models of this type are severely constrained by the simultaneous satisfaction of dark matter relic density data and by the spin-independent proton-DM scattering cross section limits in direct-detection experiments. However, such models can be viable if dark matter is multicomponent with the Higgsino-like DM contributing only a fraction of the relic density and the remainder made up from other sources. Here we discuss a two-component dark matter model where one component is the Higgsino (a Majorana fermion) of the visible sector, while the other component arises from the hidden sector and is a Dirac fermion [18]. Thus, the two-component dark matter model is a $U(1)_{X}$ extension of the standard-model gauge group where the $U(1)_{X}$ gauge boson of the hidden sector and the $U(1)_{Y}$ gauge boson of the visible sector have both kinetic [19,20] and Stueckelberg mass mixings [21-23] (for the Stueckelberg extension with an enlarged gauge group, see Refs. [24,25]). Further, the hidden sector contains matter which provides a Dirac fermion as the second component of dark matter. It is then seen that the Dirac fermion of the hidden sector provides the dominant piece of the relic density, but the Higgsino dark matter dominates the spin-independent cross section in the direct-detection experiments.

One remarkable aspect of the two-component model is the prediction of a relatively light $C P$-odd Higgs (in the range of a few hundred $\mathrm{GeV}$ ) which lies in the observable range of the future generation of colliders (see, e.g., Refs. [26,27] and $[28,29])$. Specifically, we focus here on the HighLuminosity LHC (HL-LHC) and the High-Energy LHC (HE-LHC). In this work, we carry out a detailed analysis of the integrated luminosities needed for the observation of this low-lying Higgs. Its observation would lend support to the Higgsino nature of the LSP and the multicomponent nature of dark matter. At the same time, some of the predicted spinindependent scattering cross sections also lie in the range of the next-generation dark matter direct-detection experiments. The outline of the rest of the paper is as follows: Details of the two-component model are discussed in Sec. II. The scalar sector of the theory is further elaborated in Sec. III. In Sec. IV, we give ten representative benchmarks satisfying the relic density constraint along with the Higgs boson mass constraint. An analysis of the two-component dark matter, of relic density, and of direct detection is discussed in Sec. V. Associated production of $C P$-odd Higgs along with heavy quarks at the LHC is discussed in Sec. VI, followed by the prospects of discovering a $C P$-odd Higgs at the HL-LHC and HE-LHC in Sec. VII. Conclusions are given in Sec. VIII. It is also shown that part of the parameter space of the extended model can be probed in the nextgeneration direct-detection experiments such as XENONnT and LUX-ZEPLIN.

We note in passing that there are a variety of supersymmetric $U(1)$ extensions, and their effect on DM and collider analyses have been studied extensively in the literature [30-32]. We also note that the two-component model can be easily extended to include other forms of dark matter such as an axion [33] or an ultralight axion [34-36]. Further, several works on the HL-LHC and HE-LHC discovery potential have appeared recently and in the past few years [37-42].

\section{THE MODEL}

As discussed above, we consider an extension of the standard-model gauge group by an additional Abelian gauge group $U(1)_{X}$ of gauge coupling strength $g_{X}$. The MSSM particle spectrum in the visible sector, i.e., quarks, leptons, Higgses, and their superpartners, are assumed neutral under $U(1)_{X}$. Thus, the Abelian gauge sector of the extended model contains two vector superfields-a vector superfield $B$ associated with the hypercharge gauge group $U(1)_{Y}$, and a vector superfield $C$ associated with the hidden-sector gauge group $U(1)_{X}$-and a chiral scalar superfield $S$. In the Wess-Zumino gauge, the $B$ and $C$ superfields have the following components:

$$
B=-\theta \sigma^{\mu} \bar{\theta} B_{\mu}+i \theta \theta \bar{\theta} \bar{\lambda}_{B}-i \bar{\theta} \bar{\theta} \theta \lambda_{B}+\frac{1}{2} \theta \theta \bar{\theta} \bar{\theta} D_{B}
$$

and

$$
C=-\theta \sigma^{\mu} \bar{\theta} C_{\mu}+i \theta \theta \bar{\theta} \bar{\lambda}_{C}-i \bar{\theta} \bar{\theta} \theta \lambda_{C}+\frac{1}{2} \theta \theta \bar{\theta} \bar{\theta} D_{C} .
$$

The chiral scalar superfield $S$ can be expanded in terms of its component fields as

$$
\begin{aligned}
S= & \frac{1}{2}(\rho+i a)+\theta \chi+i \theta \sigma^{\mu} \bar{\theta} \frac{1}{2}\left(\partial_{\mu} \rho+i \partial_{\mu} a\right) \\
& +\theta \theta F+\frac{i}{2} \theta \theta \bar{\theta} \bar{\sigma}^{\mu} \partial_{\mu} \chi+\frac{1}{8} \theta \theta \bar{\theta} \bar{\theta}(\square \rho+i \square a) .
\end{aligned}
$$

The gauge kinetic energy sector of the model is given by

$$
\begin{aligned}
\mathcal{L}_{\mathrm{gk}}= & -\frac{1}{4}\left(B_{\mu \nu} B^{\mu \nu}+C_{\mu \nu} C^{\mu \nu}\right)-i \lambda_{B} \sigma^{\mu} \partial_{\mu} \bar{\lambda}_{B} \\
& -i \lambda_{C} \sigma^{\mu} \partial_{\mu} \bar{\lambda}_{C}+\frac{1}{2}\left(D_{B}^{2}+D_{C}^{2}\right) .
\end{aligned}
$$

Next, we allow gauge kinetic mixing between the $U(1)_{X}$ and $U(1)_{Y}$ sectors with terms of the form 
$-\frac{\delta}{2} B^{\mu \nu} C_{\mu \nu}-i \delta\left(\lambda_{C} \sigma^{\mu} \partial_{\mu} \bar{\lambda}_{B}+\lambda_{B} \sigma^{\mu} \partial_{\mu} \bar{\lambda}_{C}\right)+\delta D_{B} D_{C}$.

As a result of Eq. (5), the hidden sector interacts with the MSSM fields via the small kinetic mixing parameter $\delta$. The kinetic terms in Eqs. (4) and (5) can be diagonalized by the transformation

$$
\left(\begin{array}{l}
B^{\mu} \\
C^{\mu}
\end{array}\right)=\left(\begin{array}{cc}
1 & -s_{\delta} \\
0 & c_{\delta}
\end{array}\right)\left(\begin{array}{l}
B^{\prime \mu} \\
C^{\prime \mu}
\end{array}\right),
$$

where $c_{\delta}=1 /\left(1-\delta^{2}\right)^{1 / 2}$ and $s_{\delta}=\delta /\left(1-\delta^{2}\right)^{1 / 2}$.

Aside from gauge kinetic mixing, we assume a Stueckelberg mass mixing between the $U(1)_{X}$ and $U(1)_{Y}$ sectors so that [21]

$$
\mathcal{L}_{\mathrm{St}}=\int d \theta^{2} d \bar{\theta}^{2}\left(M_{1} C+M_{2} B+S+\bar{S}\right)^{2} .
$$

We note that Eq. (7) is invariant under $U(1)_{Y}$ and $U(1)_{X}$ gauge transformations, so that

$$
\begin{array}{ll}
\delta_{Y} B=\Lambda_{Y}+\bar{\Lambda}_{Y}, & \delta_{Y} S=-M_{2} \Lambda_{Y}, \\
\delta_{X} C=\Lambda_{X}+\bar{\Lambda}_{X}, & \delta_{X} S=-M_{1} \Lambda_{X} .
\end{array}
$$

In component notation, $\mathcal{L}_{\mathrm{St}}$ is

$$
\begin{aligned}
\mathcal{L}_{\mathrm{St}}=- & \frac{1}{2}\left(M_{1} C_{\mu}+M_{2} B_{\mu}+\partial_{\mu} a\right)^{2}-\frac{1}{2}\left(\partial_{\mu} \rho\right)^{2} \\
& -i \chi \sigma^{\mu} \partial_{\mu} \bar{\chi}+2|F|^{2}+\rho\left(M_{1} D_{C}+M_{2} D_{B}\right) \\
& +\bar{\chi}\left(M_{1} \bar{\lambda}_{C}+M_{2} \bar{\lambda}_{B}\right)+\chi\left(M_{1} \lambda_{C}+M_{2} \lambda_{B}\right) .
\end{aligned}
$$

In the unitary gauge, the axion field $a$ is absorbed to generate mass for the $U(1)_{X}$ gauge boson. The matter sector of the model consists of the visible-sector chiral superfields, denoted by $\Phi_{i}$, where $i$ runs over all quarks, squarks, leptons, sleptons, Higgses, and Higgsino fields of the MSSM, and hidden-sector chiral superfields, denoted by $\Psi_{i}$. The Lagrangian for the matter interacting with the $U(1)$ gauge fields is given by

$$
\begin{aligned}
\mathcal{L}_{\mathrm{m}}= & \int d^{2} \theta d^{2} \bar{\theta} \sum_{i}\left[\bar{\Phi}_{i} e^{2 g_{Y} Y B+2 g_{X} X C} \Phi_{i}\right. \\
& \left.+\bar{\Psi}_{i} e^{2 g_{Y} Y B+2 g_{X} X C} \Psi_{i}\right]
\end{aligned}
$$

where $Y$ is the $U(1)_{Y}$ hypercharge and $X$ is the $U(1)_{X}$ charge. The MSSM fields are not charged under the hidden sector and vice versa, i.e., $X \Phi_{i}=0$ and $Y \Psi_{i}=0$. The minimal particle content of the hidden sector consists of a left chiral multiplet $\Psi=(\phi, f, F)$ and a charge conjugate $\Psi^{c}=\left(\phi^{\prime}, f^{\prime}, F^{\prime}\right)$ so that $\Psi$ and $\Psi^{c}$ carry opposite $U(1)_{X}$ charges and hence constitute an anomaly-free pair. The Dirac field $\psi$ formed by $f$ and $f^{\prime}$ has a mass $M_{\psi}$ arising from the term $M_{\psi} \Psi \Psi^{c}$ in the superpotential. Following SUSY breaking, the scalar fields of the hidden sector acquire soft masses equal to $m_{0}$ (the universal scalar mass of the visible sector) and the additional Dirac mass, such that

$$
m_{\phi}^{2}=m_{0}^{2}+M_{\psi}^{2}=m_{\phi^{\prime}}^{2}
$$

It is convenient from this point on to introduce Majorana spinors $\psi_{S}, \lambda_{X}$, and $\lambda_{Y}$ such that

$$
\psi_{S}=\left(\begin{array}{c}
\chi_{\alpha} \\
\bar{\chi}^{\dot{\alpha}}
\end{array}\right), \quad \lambda_{X}=\left(\begin{array}{c}
\lambda_{C \alpha} \\
\bar{\lambda}_{C}^{\dot{\alpha}}
\end{array}\right), \quad \lambda_{Y}=\left(\begin{array}{c}
\lambda_{B \alpha} \\
\bar{\lambda}_{B}^{\dot{\alpha}}
\end{array}\right)
$$

In addition to the MSSM soft-SUSY-breaking terms, we add new terms pertinent to the additional fields:

$$
\Delta \mathcal{L}_{\text {soft }}=-\left(\frac{1}{2} m_{X} \bar{\lambda}_{X} \lambda_{X}+M_{X Y} \bar{\lambda}_{X} \lambda_{Y}\right)-\frac{1}{2} m_{\rho}^{2} \rho^{2}
$$

where $m_{X}$ is the $U(1)_{X}$ gaugino mass and $M_{X Y}$ is the $U(1)_{X}-U(1)_{Y}$ mixing mass.

After electroweak symmetry breaking, $\psi_{S}$ and $\lambda_{X}$ mix with the MSSM gauginos and Higgsinos to form a $6 \times 6$ neutralino mass matrix. We choose as our basis $\left(\lambda_{Y}, \lambda_{3}\right.$, $\left.\tilde{h}_{1}, \tilde{h}_{2}, \lambda_{X}, \psi_{S}\right)$, where the last two fields arise from the extended sector and the first four, i.e., $\lambda_{Y}, \lambda_{3}, \tilde{h}_{1}, \tilde{h}_{2}$, are the gaugino and the Higgsino fields of the MSSM sector. Using Eq. (6), we rotate into the new basis $\left(\lambda_{Y}^{\prime}, \lambda_{3}, \tilde{h}_{1}, \tilde{h}_{2}, \lambda_{X}^{\prime}, \psi_{S}\right)$, so that the $6 \times 6$ neutralino mass matrix takes the form

$$
\left(\begin{array}{cccccc}
m_{1} & 0 & -c_{\beta} s_{W} M_{Z} & s_{\beta} s_{W} M_{Z} & -m_{1} s_{\delta}+M_{X Y} c_{\delta} & M_{2} \\
0 & m_{2} & c_{\beta} c_{W} M_{Z} & -s_{\beta} c_{W} M_{Z} & 0 & 0 \\
-c_{\beta} s_{W} M_{Z} & c_{\beta} c_{W} M_{Z} & 0 & -\mu & s_{\delta} c_{\beta} s_{W} M_{Z} & 0 \\
s_{\beta} s_{W} M_{Z} & -s_{\beta} c_{W} M_{Z} & -\mu & 0 & -s_{\delta} s_{\beta} s_{W} M_{Z} & 0 \\
\hline-m_{1} s_{\delta}+M_{X Y} c_{\delta} & 0 & s_{\delta} c_{\beta} s_{W} M_{Z} & -s_{\delta} s_{\beta} s_{W} M_{Z} & m_{X} c_{\delta}^{2}+m_{1} s_{\delta}^{2}-2 M_{X Y} c_{\delta} s_{\delta} & M_{1} c_{\delta}-M_{2} s_{\delta} \\
M_{2} & 0 & 0 & 0 & M_{1} c_{\delta}-M_{2} s_{\delta} & 0
\end{array}\right),
$$


where $s_{\beta} \equiv \sin \beta, c_{\beta} \equiv \cos \beta, s_{W} \equiv \sin \theta_{W}, c_{W} \equiv \cos \theta_{W}$, with $M_{Z}$ being the $Z$-boson mass. Here $m_{1}, m_{2}$ are the $U(1)_{Y}$ and $S U(2)_{L}$ gaugino masses, $\tan \beta=\left\langle H_{2}\right\rangle /\left\langle H_{1}\right\rangle$ where $H_{2}$ gives mass to the up quark and $H_{1}$ gives mass to the down quark and the lepton, and $\mu$ is the Higgs mixing parameter in the superpotential. We label the mass eigenstates as

$$
\tilde{\chi}_{1}^{0}, \tilde{\chi}_{2}^{0}, \tilde{\chi}_{3}^{0}, \tilde{\chi}_{4}^{0}, \tilde{\chi}_{5}^{0}, \tilde{\chi}_{6}^{0},
$$

where $\tilde{\chi}_{5}^{0}$ and $\tilde{\chi}_{6}^{0}$ belong to the hidden sector and mix with the usual MSSM neutralinos. In the limit of small mixings between the hidden and the MSSM sectors, the masses of the hidden-sector neutralinos are

$$
\begin{aligned}
& m_{\tilde{\chi}_{5}^{0}}=\sqrt{M_{1}^{2}+\frac{1}{4} \tilde{m}_{X}^{2}}-\frac{1}{2} \tilde{m}_{X} \quad \text { and } \\
& m_{\tilde{\chi}_{6}^{0}}=\sqrt{M_{1}^{2}+\frac{1}{4} \tilde{m}_{X}^{2}}+\frac{1}{2} \tilde{m}_{X} .
\end{aligned}
$$

We turn now to the charge neutral gauge vector boson sector. Here the $2 \times 2$ mass-squared matrix of the standard model is enlarged to become a $3 \times 3$ mass-squared matrix in the $U(1)_{X}$-extended SUGRA model. Thus, after spontaneous electroweak symmetry breaking and the Stueckelberg mass growth, the $3 \times 3$ mass-squared matrix of neutral vector bosons in the basis $\left(C_{\mu}^{\prime}, B_{\mu}^{\prime}, A_{\mu}^{3}\right)$ is given by

$$
\mathcal{M}_{V}^{2}=\left(\begin{array}{ccc}
M_{1}^{2} \kappa^{2}+\frac{1}{4} g_{Y}^{2} v^{2} s_{\delta}^{2} & M_{1} M_{2} \kappa-\frac{1}{4} g_{Y}^{2} v^{2} s_{\delta} \frac{1}{4} g_{Y} g_{2} v^{2} s_{\delta} \\
M_{1} M_{2} \kappa-\frac{1}{4} g_{Y}^{2} v^{2} s_{\delta} & M_{2}^{2}+\frac{1}{4} g_{Y}^{2} v^{2} & -\frac{1}{4} g_{Y} g_{2} v^{2} \\
\frac{1}{4} g_{Y} g_{2} v^{2} s_{\delta} & -\frac{1}{4} g_{Y} g_{2} v^{2} & \frac{1}{4} g_{2}^{2} v^{2}
\end{array}\right),
$$

where $A_{\mu}^{3}$ is the third isospin component, $g_{2}$ is the $S U(2)_{L}$ gauge coupling, $\kappa=\left(c_{\delta}-\epsilon s_{\delta}\right), \epsilon=M_{2} / M_{1}$, and $v^{2}=v_{u}^{2}+v_{d}^{2}$. The mass-squared matrix of Eq. (17) has one zero eigenvalue, which is the photon, while the other two eigenvalues are

$$
M_{ \pm}^{2}=\frac{1}{2}\left[M_{1}^{2} \kappa^{2}+M_{2}^{2}+\frac{1}{4} v^{2}\left[g_{Y}^{2} c_{\delta}^{2}+g_{2}^{2}\right] \pm \sqrt{\left(M_{1}^{2} \kappa^{2}+M_{2}^{2}+\frac{1}{4} v^{2}\left[g_{Y}^{2} c_{\delta}^{2}+g_{2}^{2}\right]\right)^{2}-\left[M_{1}^{2} g_{2}^{2} v^{2} \kappa^{2}+M_{1}^{2} g_{Y}^{2} v^{2} c_{\delta}^{2}+M_{2}^{2} g_{2}^{2} v^{2}\right]}\right]
$$

where $M_{+}$is identified as the $Z^{\prime}$-boson mass, while $M_{-}$is the $Z$-boson mass. The diagonalization of the mass-squared matrix of Eq. (17) can be done via two orthogonal transformations, where the first is given by [23]

$$
\mathcal{O}=\left(\begin{array}{ccc}
1 / c_{\delta} & -s_{\delta} / c_{\delta} & 0 \\
s_{\delta} / c_{\delta} & 1 / c_{\delta} & 0 \\
0 & 0 & 1
\end{array}\right)
$$

which transforms the mass matrix to $\mathcal{M}_{V}^{\prime 2}=\mathcal{O}^{T} \mathcal{M}_{V}^{2} \mathcal{O}$,

$$
\mathcal{M}_{V}^{\prime 2}=\left(\begin{array}{ccc}
M_{1}^{2} & M_{1}^{2} \epsilon^{\prime} & 0 \\
M_{1}^{2} \epsilon^{\prime} & M_{1}^{2} \epsilon^{\prime 2}+\frac{1}{4} g_{Y}^{2} v^{2} c_{\delta}^{2} & -\frac{1}{4} g_{Y} g_{2} v^{2} c_{\delta} \\
0 & -\frac{1}{4} g_{Y} g_{2} v^{2} c_{\delta} & \frac{1}{4} g_{2}^{2} v^{2}
\end{array}\right)
$$

where $\epsilon^{\prime}=\epsilon c_{\delta}-s_{\delta}$. The gauge eigenstates of $\mathcal{M}_{V}^{\prime 2}$ can be rotated into the corresponding mass eigenstates $\left(Z^{\prime}, Z, \gamma\right)$ using the second transformation via the rotation matrix

$\mathcal{R}=\left(\begin{array}{ccc}c_{\eta} c_{\phi}-s_{\theta} s_{\phi} s_{\eta} & s_{\eta} c_{\phi}+s_{\theta} s_{\phi} c_{\eta} & -c_{\theta} s_{\phi} \\ c_{\eta} s_{\phi}+s_{\theta} c_{\phi} s_{\eta} & s_{\eta} s_{\phi}-s_{\theta} c_{\phi} c_{\eta} & c_{\theta} c_{\phi} \\ -c_{\theta} s_{\eta} & c_{\theta} c_{\eta} & s_{\theta}\end{array}\right)$

with $c_{\eta}\left(c_{\theta}\right)\left(c_{\phi}\right) \equiv \cos \eta(\cos \theta)(\cos \phi)$ and $s_{\eta}\left(s_{\theta}\right)\left(s_{\phi}\right) \equiv$ $\sin \eta(\sin \theta)(\sin \phi)$, where $\eta$ represents the mixing angle between the new gauge sector and the standard-model gauge bosons, while the other angles are given by

$$
\tan \phi=\epsilon^{\prime}, \quad \tan \theta=\frac{g_{Y}}{g_{2}} c_{\delta} \cos \phi,
$$

such that $\mathcal{R}^{T} \mathcal{M}_{V}^{\prime 2} \mathcal{R}=\operatorname{diag}\left(M_{Z^{\prime}}^{2}, M_{Z}^{2}, 0\right)$. The resulting mixing angle is thus given by

$$
\tan 2 \eta \simeq \frac{2 \epsilon^{\prime} M_{Z}^{2} \sin \theta}{M_{Z^{\prime}}^{2}-M_{Z}^{2}+\left(M_{Z^{\prime}}^{2}+M_{Z}^{2}-M_{W}^{2}\right) \epsilon^{\prime 2}},
$$

with $M_{W}=g_{2} v / 2, M_{Z^{\prime}} \equiv M_{+}$and $M_{Z} \equiv M_{-}$.

\section{THE SCALAR SECTOR OF THE $U(1)_{X^{-}}$ EXTENDED MSSM/SUGRA}

The addition of the chiral scalar superfield $S$ and the hidden-sector matter fields brings about new scalar fields to the theory. Thus, the scalar fields of the $U(1)_{X}$-extended MSSM/SUGRA are the Higgs fields, the scalar $\rho$, and the fields $\phi$ and $\phi^{\prime}$ of the hidden sector. In the MSSM, the Higgs sector contains two Higgs doublets $H_{d}$ and $H_{u}$,

$$
H_{d}=\left(\begin{array}{c}
H_{d}^{0} \\
H_{d}^{-}
\end{array}\right) \quad \text { and } \quad H_{u}=\left(\begin{array}{c}
H_{u}^{+} \\
H_{u}^{0}
\end{array}\right),
$$

with opposite hypercharge, which ensures the cancellation of chiral anomalies. Here $H_{d}$ gives mass to the down-type 
quarks and the leptons, while $H_{u}$ gives mass to up-type quarks. The Higgs potential in the MSSM arises from three sources: the $F$ term of the superpotential; the $D$ terms containing the quartic Higgs interaction and the soft-SUSYbreaking Higgs mass squared, $m_{H_{d}}^{2}$ and $m_{H_{u}}^{2}$; and the bilinear $B$ term. The additional scalar field $\rho$ enters the Higgs potential and mixes with the MSSM Higgs doublets. The full $C P$-conserving Higgs scalar potential in the extended model can be written as

$$
\begin{aligned}
V_{H}= & {\left[|\mu|^{2}+m_{H_{d}}^{2}-\frac{1}{2} g_{Y} \rho M_{1}\left(\epsilon-s_{\delta}\right)\right]\left|H_{d}\right|^{2} } \\
& +\left[|\mu|^{2}+m_{H_{u}}^{2}+\frac{1}{2} g_{Y} \rho M_{1}\left(\epsilon-s_{\delta}\right)\right]\left|H_{u}\right|^{2} \\
& -B \epsilon_{i j}\left(H_{u}^{i} H_{d}^{j}+\text { H.c. }\right)+\left(\frac{g_{Y}^{2} c_{\delta}^{2}+g_{2}^{2}}{8}\right) \\
& \times\left(\left|H_{d}\right|^{2}-\left|H_{u}\right|^{2}\right)^{2}+\frac{1}{2} g_{2}^{2}\left|H_{d}^{\dagger} H_{u}\right|^{2} \\
& +\frac{1}{2}\left(M_{1}^{2}+M_{2}^{2}+m_{\rho}^{2}\right) \rho^{2}+\Delta V_{\text {loop }},
\end{aligned}
$$

where $\mu$ is the Higgs mixing parameter appearing in the superpotential term $\mu \hat{H}_{u} \cdot \hat{H}_{d}$. The neutral components of the Higgs doublets and the scalar $\rho$ can be expanded around their vacuum expectation values (VEVs) so that

$$
\begin{aligned}
H_{d}^{0} & =\frac{1}{\sqrt{2}}\left(v_{d}+\phi_{d}+i \psi_{d}\right), \\
H_{u}^{0} & =\frac{1}{\sqrt{2}}\left(v_{u}+\phi_{u}+i \psi_{u}\right), \\
\rho & =v_{\rho}+\phi_{\rho} .
\end{aligned}
$$

The MSSM $2 \times 2$ Higgs mass matrix is now extended to become $3 \times 3$ with the new scalar field $\phi_{\rho}$ mixing with the two $C P$-even Higgs fields $\phi_{d}$ and $\phi_{u}$. As a result, the masses of the $C P$-even Higgses $h$ and $H$ are modified by amounts proportional to $\epsilon$ and $\delta$, which, however, are small. Similarly, the corrections to the $C P$-odd Higgs mass induced by the new sector are negligible. Minimizing the Higgs potential of Eq. (25) in the $\phi_{d}, \phi_{u}$, and $\phi_{\rho}$ directions, we obtain the constraints

$$
\begin{aligned}
m_{H_{d}}^{2}+\mu^{2}-B \tan \beta+\frac{1}{2} M_{Z}^{2} \cos 2 \beta+\Delta_{\mathrm{St}} & =0, \\
m_{H_{u}}^{2}+\mu^{2}-B \cot \beta-\frac{1}{2} M_{Z}^{2} \cos 2 \beta-\Delta_{\mathrm{St}} & =0, \\
\left(M_{1}^{2}+M_{2}^{2}+m_{\rho}^{2}\right) v_{\rho}-\frac{1}{4} g_{Y} v^{2} M_{1}\left(\epsilon-s_{\delta}\right) \cos 2 \beta & =0,
\end{aligned}
$$

where

$$
\Delta_{\mathrm{St}}=-\frac{1}{2} g_{Y} v_{\rho} M_{1}\left(\epsilon-s_{\delta}\right)+\frac{1}{8} v^{2} g_{Y}^{2} s_{\delta}^{2} \cos 2 \beta .
$$

The last line of Eq. (27) gives

$$
v_{\rho}=\frac{g_{Y} v^{2} \cos 2 \beta}{4\left(M_{1}^{2}+M_{2}^{2}+m_{\rho}^{2}\right)} M_{1}\left(\epsilon-s_{\delta}\right),
$$

which is typically small, since $\epsilon$ and $\delta$ are small.

\section{IV. $U(1)_{X}$-EXTENDED MSSM/SUGRA BENCHMARKS}

The particle content of the $U(1)_{X}$-extended MSSM/ SUGRA model discussed in Secs. II and III consists of the particles of the MSSM, and from the hidden sector, three spin- 0 particles $\left(\rho, \phi, \phi^{\prime}\right)$, three spin- $1 / 2$ particles (a Dirac fermion $\psi$ and two Majorana neutralinos $\tilde{\chi}_{5}^{0}, \tilde{\chi}_{6}^{0}$ ), and one massive vector boson $Z^{\prime}$. The model is implemented in the Mathematica package SARAH V4.14.1 $[43,44]$, which generates model files for SPheno-4 - 0.3 [45,46], which in turn produces the sparticle spectrum and CalcHep/CompHep $[47,48]$ files used by micrOMEGAs - 5 . 0.4 [49] to determine the dark matter relic density and UFO files which are input to MadGraph5 [50]. The input parameters of the $U(1)_{X}$-extended MSSM/ SUGRA [51] with hidden-sector matter are taken to be $m_{0}$, $A_{0}, m_{1}, m_{2}, m_{3}, \tan \beta, \operatorname{sgn}(\mu), M_{1}, m_{X}, M_{\psi}, B_{\psi}, \delta$, and $g_{X}$, where $m_{0}, A_{0}, m_{3}, B_{\psi}$ are the universal scalar mass, the universal trilinear coupling, $S U(3)_{c}$ gaugino mass, and $B_{\psi}$ is the bilinear parameter of the Dirac mass term in the superpotential; all of these are taken to be at the GUT scale. Note that all other parameters listed above have already been defined in the previous sections.

Table I shows ten representative benchmarks covering a mass range of the $C P$-odd Higgs from $\sim 300 \mathrm{GeV}$ to $750 \mathrm{GeV}$. The value of $\mu$ from the table is $\mathcal{O}(100) \mathrm{GeV}$, which arises naturally on the hyperbolic branch and supports the argument for naturalness. Since the mass of the $C P$-odd Higgs depends on $\mu B$, one expects light $C P$-odd states. It is to be noted that a larger fraction of the relic density is contributed by the Dirac fermion of the hidden sector, while the Higgsino-like neutralinos contribute a smaller fraction, which is due to the fact that $\mu$ is small. So, having a two-component DM model respecting naturalness leads to a light $C P$-odd Higgs.

The $C P$-odd Higgs mass along with the neutralino, chargino, stop, gluino, and stau masses are presented in Table II. We also show the mass of the lightest $C P$ even Higgs consistent with the observed $125 \mathrm{GeV}$ Higgs within $\pm 2 \mathrm{GeV}$ error. In some of those benchmarks, the value of $m_{0}$ is quite small-for instance, point ( $\mathrm{g}$ ) has $m_{0} \sim 800 \mathrm{GeV}$, while the stop mass is $\sim 5 \mathrm{TeV}$. The reason for this is the large value of $m_{3}$, which via the RGE running generates squark masses in the several-TeV range [52]. 
TABLE I. Input parameters for the benchmarks used in this analysis. Here $M_{X Y}=0=B$ at the GUT scale, and $M_{2}$ is chosen at the GUT scale, so that it is nearly vanishing at the electroweak scale. All masses are in GeV.

\begin{tabular}{|c|c|c|c|c|c|c|c|c|c|c|c|c|c|}
\hline Model & $m_{0}$ & $A_{0}$ & $m_{1}$ & $m_{2}$ & $m_{3}$ & $\mu$ & $M_{1}$ & $m_{X}$ & $M_{\psi}$ & $B_{\psi}$ & $\tan \beta$ & $g_{X}$ & $\delta$ \\
\hline (a) & 8115 & -7477 & 6785 & 9115 & 4021 & 423 & 1261 & 27 & 627 & 9283 & 6 & 0.06 & 0.02 \\
\hline (b) & 1743 & 898 & 4551 & 2160 & 4084 & 301 & -1086 & 27 & 627 & 5167 & 10 & 0.07 & 0.02 \\
\hline (c) & 1056 & -920 & 1706 & 3417 & 3396 & 243 & 1059 & 89 & 525 & 2846 & 10 & 0.03 & 0.01 \\
\hline (d) & 8424 & -2488 & 6165 & 3544 & 2466 & 330 & -1469 & 473 & 733 & 4680 & 12 & 0.03 & 0.01 \\
\hline (e) & 2011 & -2462 & 3008 & 5030 & 3833 & 598 & 875 & 38 & 425 & 3248 & 9 & 0.06 & 0.06 \\
\hline (f) & 4637 & -4045 & 7004 & 5480 & 2727 & 511 & -1230 & 372 & 613 & 7557 & 15 & 0.04 & 0.04 \\
\hline (g) & 819 & 477 & 7847 & 1218 & 3040 & 201 & 820 & 509 & 401 & 3425 & 12 & 0.05 & 0.09 \\
\hline (h) & 3881 & -2580 & 7449 & 4870 & 4429 & 268 & 850 & 152 & 419 & 9199 & 13 & 0.08 & 0.02 \\
\hline (i) & 1349 & -2722 & 3938 & 4420 & 2558 & 482 & 1292 & 19 & 636 & 4235 & 15 & 0.07 & 0.08 \\
\hline (j) & 2015 & -4435 & 2695 & 5399 & 2470 & 217 & 1343 & 690 & 670 & 4587 & 11 & 0.03 & 0.03 \\
\hline
\end{tabular}

With heavy gluinos and stops, experimental limits on their masses from ATLAS and CMS can be evaded. Also, the LSP and chargino masses presented in Table II have not yet been ruled out by experiment.

Similar MSSM benchmark scenarios have appeared in Ref. [53], where heavy Higgses can be probed at the LHC using an effective field theory approach with a spectrum containing light charginos and neutralinos, while the rest of the SUSY particles are heavy.

The analysis given here is within the framework of SUGRA, which is a high-scale model based on supersymmetry and supergravity. Within the framework of SUGRA, the number of inputs is significantly reduced relative to low scales, where one will need around 105 parameters to describe the soft sector. We note in passing that the extra $U(1)$ is from the hidden sector and does not arise from a unified visible-sector gauge group such as $S U(5)$ or $S O(10)$, from which the standard-model gauge group originates. So its coupling $g_{X}$ is not constrained to be unified with the rest of the gauge group couplings. While $g_{X}$ is not constrained by unification, it is constrained by experiment in that the limit on it from collider experiments must be satisfied as discussed below. Thus, the particle spectrum of the model contains an extra neutral massive gauge boson, $Z^{\prime}$. Stringent constraints are set on the mass of the $Z^{\prime}$ [54] and most recently by ATLAS [55] using
$139 \mathrm{fb}^{-1}$ of data. In new physics models containing $Z^{\prime}$ with SM couplings, the mass limit is set at $m_{Z^{\prime}}>5.1 \mathrm{TeV}$. For a model with an extra $U(1)_{X}$ with a gauge coupling strength $g_{X}$, the limit can be written as

$$
\frac{m_{Z^{\prime}}}{g_{X}} \gtrsim 12 \mathrm{TeV} .
$$

For the benchmarks of Table I, the $Z^{\prime}$ mass obtained from Eq. (18) is $\sim M_{1}$, since $M_{2} \sim 0$ and $s_{\delta} \ll 1$. Thus, the spectrum contains a $Z^{\prime}$ with a mass range of $\sim 800 \mathrm{GeV}$ to $\sim 1500 \mathrm{GeV}$. However, since the $U(1)_{X}$ coupling $g_{X}<0.1$, the limit of Eq. (30) is satisfied for all the benchmarks. The smallness of $g_{X}$ also means that the $Z^{\prime}$ coupling to SM particles is tiny; therefore its production cross section at $p p$ colliders is suppressed, and thus a $Z^{\prime}$ in the mass range noted above is consistent with the current experimental constraints. Further, from Eq. (18), the $Z$-boson mass receives a correction due to gauge kinetic and mass mixings. Knowing that $M_{2} \ll M_{1}$ and $s_{\delta} \ll 1$, we can write $M_{-}^{2}$ as

$$
M_{-}^{2} \simeq M_{Z}^{2}+\frac{\epsilon}{2} g_{Y}^{2} v^{2} \frac{s_{\delta}}{c_{\delta}}+\frac{1}{4} g_{2}^{2} v^{2}\left(\frac{\epsilon}{\kappa}\right)^{2}
$$

For the benchmarks, $\epsilon$ takes values in the range $\mathcal{O}\left(10^{-4}\right)-$ $\mathcal{O}\left(10^{-3}\right)$ with $\kappa \sim 1$, and the correction to the $Z$-boson mass falls within the current experimental error bars.

TABLE II. Display of the SM-like Higgs boson mass, the stau mass, the relevant electroweak gaugino masses, the $C P$-odd Higgs

\begin{tabular}{|c|c|c|c|c|c|c|c|c|c|c|c|}
\hline Model & $h$ & $\tilde{\chi}_{1}^{0}$ & $\tilde{\chi}_{1}^{ \pm}$ & $\tilde{\tau}$ & $\tilde{\chi}_{5}^{0}$ & $\tilde{t}$ & $\tilde{g}$ & A & $\Omega h^{2}$ & $\left(\Omega h^{2}\right)_{\chi}$ & $\left(\Omega h^{2}\right)_{\psi}$ \\
\hline (a) & 123.3 & 455.9 & 457.1 & 8109 & 1245 & 6343 & 8408 & 305.8 & 0.124 & 0.022 & 0.102 \\
\hline (b) & 123.3 & 322.6 & 324.9 & 2115 & 1008 & 5898 & 8195 & 351.8 & 0.101 & 0.012 & 0.089 \\
\hline (c) & 123.1 & 258.9 & 262.6 & 665.6 & 1015 & 4565 & 6855 & 408.9 & 0.116 & 0.009 & 0.107 \\
\hline (d) & 124.0 & 354.8 & 356.4 & 8425 & 1250 & 6573 & 5467 & 450.8 & 0.117 & 0.019 & 0.098 \\
\hline (e) & 123.9 & 639.5 & 642.2 & 1875 & 851.5 & 4943 & 7712 & 504.2 & 0.106 & 0.042 & 0.064 \\
\hline (f) & 124.7 & 544.3 & 545.7 & 4982 & 1055 & 4314 & 5803 & 547.3 & 0.125 & 0.031 & 0.094 \\
\hline (g) & 123.1 & 212.4 & 215.3 & 1906 & 601.8 & 4646 & 6229 & 604.2 & 0.118 & 0.006 & 0.112 \\
\hline (h) & 125.0 & 289.1 & 290.5 & 4426 & 775.5 & 6109 & 8565 & 650.9 & 0.121 & 0.009 & 0.112 \\
\hline (i) & 124.3 & 510.8 & 512.9 & 1627 & 1276 & 3077 & 5292 & 702.7 & 0.118 & 0.028 & 0.090 \\
\hline (j) & 125.0 & 231.5 & 233.7 & 1845 & 1041 & 2335 & 5164 & 750.3 & 0.113 & 0.008 & 0.105 \\
\hline
\end{tabular}
mass, and the relic density for the benchmarks of Table I computed at the electroweak scale. All masses are in GeV. 


\section{TWO-COMPONENT DARK MATTER AND ITS DIRECT DETECTION}

As noted earlier, one of the constraints on dark matter models is the relic density constraint, which, according to the Planck Collaboration [10], is given by

$$
\left(\Omega h^{2}\right)_{\text {PLANCK }}=0.1198 \pm 0.0012 .
$$

Since there are two components to dark matter, the total relic density is the sum of the neutralino and the Dirac fermion relic densities, i.e.,

$$
\left(\Omega h^{2}\right)_{\mathrm{DM}}=\left(\Omega h^{2}\right)_{\chi}+\left(\Omega h^{2}\right)_{\psi} .
$$

Further, the spin-independent DM-proton cross section that enters in direct-detection experiments is given by

$$
\sigma_{\mathrm{DM}-p}^{\mathrm{SI}}=R_{\chi} \sigma_{p \chi}^{\mathrm{SI}}+R_{\psi} \sigma_{p \psi}^{\mathrm{SI}},
$$

where

$$
\begin{aligned}
& \mathcal{R}_{\chi}=\left(\Omega h^{2}\right)_{\chi} /\left(\Omega h^{2}\right)_{\text {PLANCK }}, \quad \text { and } \\
& \mathcal{R}_{\psi}=\left(\Omega h^{2}\right)_{\psi} /\left(\Omega h^{2}\right)_{\text {PLANCK }} .
\end{aligned}
$$

Thus, one finds that not only the sum of the neutralino and the Dirac fermion relic densities, but also their individual contributions have observable consequences, as seen from Eq. (34).

The main processes that enter in the neutralino and Dirac fermion relic abundance are

$$
\begin{aligned}
& \chi \chi \leftrightarrow \text { SM SM } \\
& \psi \bar{\psi} \leftrightarrow \text { SM SM } \\
& \psi \bar{\psi} \leftrightarrow \chi \chi .
\end{aligned}
$$

Note that the process $\chi \bar{\psi} \leftrightarrow$ SM SM cannot happen, since the only allowed vertex is $\psi \chi \phi$, and $\phi$ does not couple to
SM particles. In order to calculate the relic density in the two-component model, one must solve the Boltzmann equations for the $\chi$ and $\psi$ number densities $n_{\chi}$ and $n_{\psi}$. Taking into consideration the processes in Eq. (36), the coupled Boltzmann equations for $n_{\chi}$ and $n_{\psi}$ are [18]

$$
\begin{aligned}
\frac{d n_{\chi}}{d t}= & -3 H n_{\chi}-\langle\sigma v\rangle_{\chi \chi}\left(n_{\chi}^{2}-n_{\chi, \mathrm{eq}}^{2}\right) \\
& +\frac{1}{2}\langle\sigma v\rangle_{\psi \bar{\psi} \rightarrow \chi \chi}\left(n_{\psi}^{2}-n_{\psi, \mathrm{eq}}^{2}\right), \\
\frac{d n_{\psi}}{d t}= & -3 H n_{\psi}-\frac{1}{2}\langle\sigma v\rangle_{\psi \bar{\psi}}\left(n_{\psi}^{2}-n_{\psi, \mathrm{eq}}^{2}\right),
\end{aligned}
$$

where $\langle\sigma v\rangle_{\chi \chi}$ denotes $\langle\sigma v\rangle_{\chi \chi \rightarrow \mathrm{SM} \mathrm{SM}},\langle\sigma v\rangle_{\psi \psi \psi}$ refers to $\psi \bar{\psi} \rightarrow$ SMSM, $\chi \chi$ and $n_{\mathrm{eq}}$ represents the equilibrium number density. The factor of $1 / 2$ appearing in some of the terms is due to the fact that $\psi$ is a Dirac fermion. With the exception of point (e) in Table II, $M_{\psi}>M_{\tilde{\chi}_{1}^{0}}$, and so $\psi$ freezes out earlier, i.e., at a higher temperature, $T_{f}$, than $\tilde{\chi}_{1}^{0}$. Notice the small mass gap between $\tilde{\chi}_{1}^{0}$ and $\tilde{\chi}_{1}^{ \pm}$, resulting in the activation of the coannihilation channel. The solution to Eq. (37) does not have a closed form and must be solved numerically. The total relic density can be expressed as

$$
\Omega h^{2} \simeq \frac{C_{\chi}}{\int_{0}^{x_{f}^{\chi}}\langle\sigma v\rangle_{\chi \chi} d x}+\frac{C_{\psi}}{\int_{0}^{x_{f}^{\psi \prime}}\langle\sigma v\rangle_{\psi \bar{\psi}} d x},
$$

where $x_{f}=m / T_{f}$ ( $m$ being the mass of the DM particle)

\begin{tabular}{|c|c|c|c|c|c|}
\hline Model & $\begin{array}{c}\langle\sigma v\rangle_{\chi \chi \rightarrow \mathrm{SM} \mathrm{SM}} \\
\times 10^{-26}\left[\mathrm{~cm}^{3} / \mathrm{s}\right]\end{array}$ & $\begin{array}{c}\langle\sigma v\rangle_{\psi \psi \psi \rightarrow \mathrm{SM} \mathrm{SM}} \\
\times 10^{-28}\left[\mathrm{~cm}^{3} / \mathrm{s}\right]\end{array}$ & $\begin{array}{c}\langle\sigma v\rangle_{\psi \psi \psi \rightarrow \chi \chi} \\
\times 10^{-31}\left[\mathrm{~cm}^{3} / \mathrm{s}\right]\end{array}$ & $\begin{array}{c}\mathcal{R}_{\chi} \times \sigma_{p \tilde{\chi}_{1}^{0}}^{\mathrm{SI}} \\
\times 10^{-47}\left[\mathrm{~cm}^{2}\right]\end{array}$ & $\begin{array}{c}\mathcal{R}_{\psi} \times \sigma_{p \psi}^{\mathrm{SI}} \\
\times 10^{-50}\left[\mathrm{~cm}^{2}\right]\end{array}$ \\
\hline (a) & 4.68 & 5.55 & 2.17 & 1.09 & 0.19 \\
\hline (b) & 9.62 & 0.51 & 34.3 & 6.86 & 903.4 \\
\hline (c) & 13.5 & 0.03 & 2.51 & 5.63 & 73.2 \\
\hline (d) & 7.68 & 141 & 8380 & 2.77 & 30.0 \\
\hline (e) & 2.59 & 1.65 & 0.01 & 10.67 & 2.41 \\
\hline (f) & 3.37 & 698 & 3440 & 2.04 & 1.30 \\
\hline (g) & 19.4 & 1.23 & 0.86 & 5.47 & 9.79 \\
\hline (h) & 11.1 & 0.69 & 5.47 & 0.52 & 32.69 \\
\hline (i) & 3.82 & 3.23 & 0.33 & 3.63 & 0.96 \\
\hline (j) & 16.5 & 18.6 & 1020 & 0.93 & 145.5 \\
\hline
\end{tabular}
and the $C$ 's are constants proportional to $g^{*^{-1 / 2}} M_{\mathrm{Pl}}^{-1}$, with $g^{*}$ being the effective number of degrees of freedom at freezeout for $\chi$ or $\psi$, and $M_{\mathrm{Pl}}$ being the Planck mass. In columns 2-4 of Table III, we give the size of the thermally averaged annihilation cross sections of the processes in Eq. (36). The largest cross section is that of $\chi \chi \leftrightarrow$ SM SM, since it has weak-scale couplings, while reactions involving $\psi$ have cross sections that are 2 to 5 orders of magnitude less than those of $\chi \chi$. The reason is that $\psi \bar{\psi} \bar{s}$ annihilation proceeds

TABLE III. The thermally averaged annihilation cross sections, $\langle\sigma v\rangle_{\chi \chi \rightarrow \mathrm{SM} \mathrm{SM}},\langle\sigma v\rangle_{\psi \psi \psi \rightarrow \mathrm{SM} \mathrm{SM}}$, and $\langle\sigma v\rangle_{\psi \psi \psi \rightarrow \chi \chi}$, and the spin-independent proton-DM scattering cross-sections $\mathcal{R}_{\chi} \times \sigma_{p \tilde{\chi}_{1}^{0}}^{\mathrm{SI}}$ and $\mathcal{R}_{\psi} \times \sigma_{p \psi}^{\mathrm{SI}}$ for the benchmarks of Table I. 


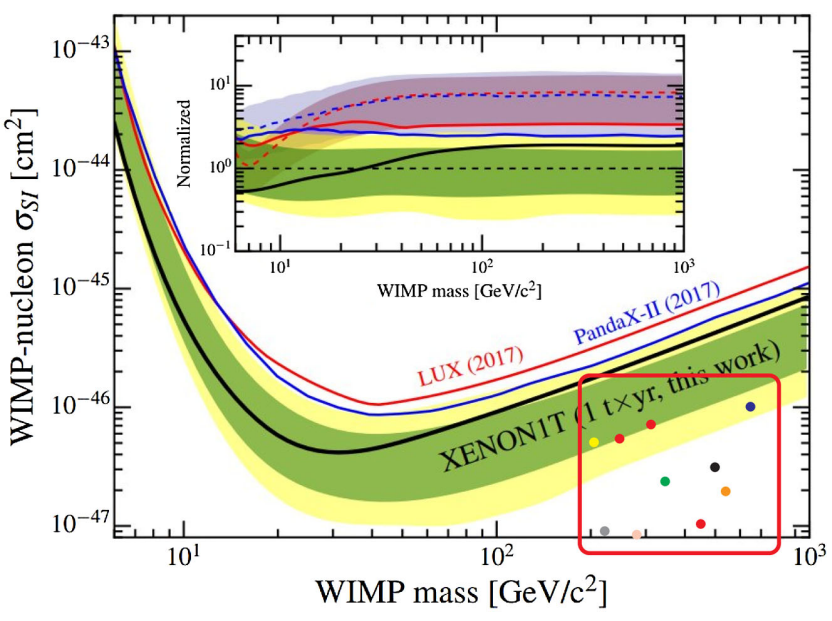

FIG. 1. The SI proton-neutralino cross section exclusion limits as a function of the LSP mass from XENON1T (taken from Ref. [56]). The ten benchmarks are overlaid on the plot, showing them lying below the upper limit (black curve). The inset shows the limits from LUX 2017 [58], PandaX-II [59], and XENON1T along with the uncertainty bands normalized to the sensitivity median defined in Ref. [56].

through an $s$-channel exchange of $\gamma, Z, Z^{\prime}$ with couplings proportional to $g_{X}, s_{\delta}$, and $\epsilon$. The smallness of these parameters renders the cross section tiny.

We turn now to the spin-independent (SI) proton-DM scattering cross section in direct-detection experiments. The main contribution to the proton-neutralino scattering cross section comes from a $t$-channel exchange of a Higgs boson $(h / H)$, while the proton-Dirac fermion scattering cross section involves a box diagram with the exchange of the scalar $\phi$ from the hidden sector, which explains its small value (last column in Table III) compared to the protonneutralino one (fifth column in Table III). To get an idea of where the benchmarks lie relative to the current experimental sensitivity of direct-detection experiments, we plot in Fig. 1 the ten benchmarks along with the most recent experimental limits from XENON1T [56] for the protonneutralino cross section (the color code for those points has no meaning here). We note that some of these benchmarks may be accessible in future experiments such as XENONnT and LUX-ZEPLIN [57]. Note that for most of the benchmarks, $\mathcal{R}_{\psi} \times \sigma_{p \psi}^{\mathrm{SI}}$ is very small and lies well below the sensitivity limit of future experiments, while others lie even below the neutrino floor.

\section{ASSOCIATED PRODUCTION OF CP-ODD HIGGS WITH HEAVY QUARKS}

As a result of electroweak symmetry breaking and the Stueckelberg mass growth, the Higgs sector of the $U(1)_{X^{-}}$ extended MSSM has six degrees of freedom corresponding to three $C P$-even Higgs, $h, H$, and $\rho$; one $C P$-odd Higgs, $A$; and two charged Higgs, $H^{ \pm}$. In this section, we discuss the production and decay of the $C P$-odd Higgs $A$. Since the weak scale of supersymmetry is high, lying in the few-TeV region, we are in the so-called decoupling limit, where the light $C P$-even Higgs $h$ is SM-like, and $H, A$, and $H^{ \pm}$ (charged Higgs) have comparable masses much greater than $h$. In this case, $A$ exhibits no tree-level couplings to the gauge bosons or couplings to down-type (up-type) fermions that are (inversely) proportional to $\tan \beta$. For high $\tan \beta$ values, $\tan \beta \gtrsim 10$, the $H, A$ Yukawa couplings to bottom quarks and tau leptons are strongly enhanced, while those to top quarks are strongly suppressed. In this region, the $b$ quark will play an important role as its coupling to the $C P$-odd Higgs is enhanced. For this reason, we examine the associated production of $A$ with bottomantibottom quarks, $b \bar{b} A$.

There are two approaches to calculating the production cross section of $A$ in association with $b \bar{b}$. The first considers the $b$ quark to be heavy and appearing only in the final state, as shown in Fig. 2, where the leading-order (LO) partonic processes are

$$
g g \rightarrow b \bar{b} A, \quad q \bar{q} \rightarrow b \bar{b} A .
$$

This approach constitutes the four-flavor scheme (4FS), in which the mass of the $b$ quark is considered part of the hard scale of the process. Apart from its dependence on the $C P$-odd Higgs mass and $\tan \beta$, the cross section is sensitive to the $b$-quark mass, which is taken to be the running mass at the appropriate renormalization and factorization scales. The LO $2 \rightarrow 3$ diagrams in the $4 \mathrm{FS}$ begin at $\mathcal{O}\left(\alpha_{S}^{2}\right)$, while the next-to-leading-order (NLO) diagrams (bottom three diagrams of Fig. 2) contain bottom and top quarks circulating in the loops. Here the LO cross section is proportional to $\alpha_{S}^{2} y_{b}^{2}$, while at NLO, the cross section is proportional to $\alpha_{S}^{3} y_{b}^{2}$ and $\alpha_{S}^{3} y_{b} y_{t}$, where $y_{b}, y_{t}$ are the bottom and top Yukawa couplings, and where the $y_{b} y_{t}$ term corresponds to interference between the gluon-fusion (with a top quark in the loop) and $b \bar{b} A$ processes. As mentioned before, the $C P$-odd Higgs coupling to the top quark is suppressed, and thus the diagrams involving top quarks do not contribute significantly to the cross section. Following the prescription of Ref. [60], the hard scale of the process is chosen at the renormalization and factorization scales such that $\mu_{R}=\mu_{F}=\left(m_{A}+2 m_{b}\right) / 4$, with $m_{A}$ being the $C P$-odd Higgs mass and $m_{b}$ being the $b$-quark pole mass, while the running $b$-quark mass is $\bar{m}_{b}\left(\mu_{F}\right)$ (see Table IV). The $4 \mathrm{FS}$ NLO cross section at fixed order in $\alpha_{S}$ is calculated with MadGraph5_aMC@NLO-2.6.3 using FeynRules [61] UFO files [62,63] for the type-II two-Higgs-doublet model (2HDM). The choice of the latter is justified due to the fact that SUSY-QCD effects for our benchmarks are very minimal, since the squarks and gluinos are heavy. The cross sections at $14 \mathrm{TeV}$ and $27 \mathrm{TeV}$ are displayed in Table IV along with uncertainties arising from scale variations. 

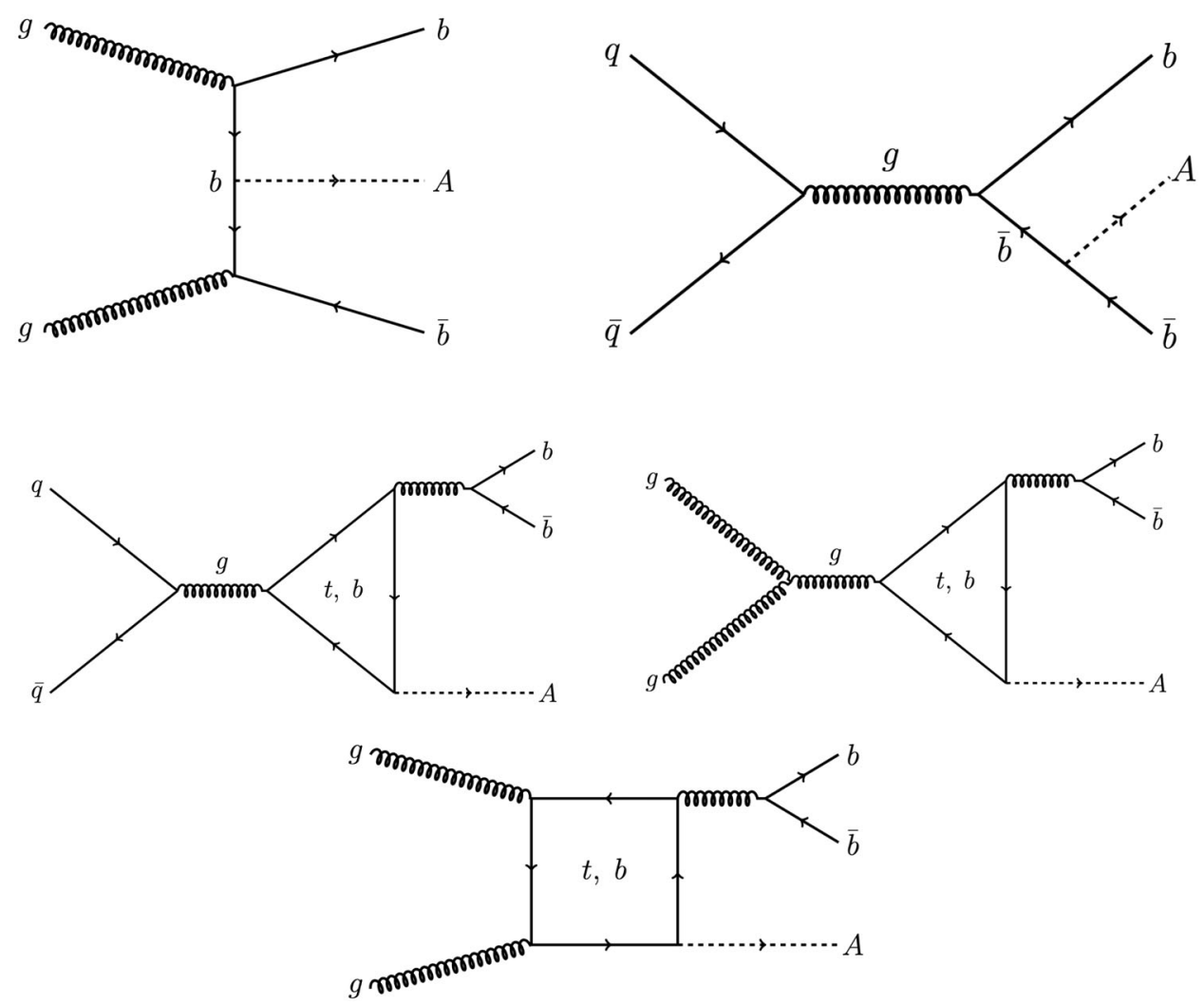

FIG. 2. A sample of the tree (top two) and one-loop (bottom three) Feynman diagrams for $b \bar{b} A$ production at the LHC in the fourflavor scheme.

At any order in perturbation, the 4FS cross section involves terms $\sim \alpha_{S}^{n} \log ^{n}\left(\mu_{F} / m_{b}\right)$ resulting from collinear splitting of gluons to $b \bar{b}$ pairs. This term is kept under control as long as $\mu_{F} \sim m_{b}$; however, this is not the case for our benchmarks, especially for larger masses of the $C P$-odd Higgs, where such a term spoils perturbative convergence. The way to resolve this issue is by absorbing those terms to all orders in $\alpha_{S}$. The resummation of those potentially large logarithms is done via the DGLAP evolution of $b$-quark PDFs, which constitutes the second approach to calculating cross sections, which is the five-flavor scheme (5FS). In this scheme, $b$ quarks are massless and considered as partons, so they do not appear in the final states at the partonic level. Hence, the LO process (zeroth order in $\alpha_{S}$ ) in the 5FS for $C P$-odd Higgs production is

$$
b \bar{b} \rightarrow A .
$$

At the parton level, the 4FS approach has the advantage over that of the 5FS, since realistic $b$ tagging can be done with the former, while the latter does not possess this property due to less rich final states. However, the 5FS parton-level events are matched to parton showers, which add $b$ jets allowing proper $b$ tagging at the analysis level. This is, of course, pertinent to LO calculations, while at higher orders in QCD, the 5FS starts to exhibit richer final states with the appearance of $b$ quarks.
The 5FS $b \bar{b} A$ production cross section is known at next-toNLO (NNLO), and we use SusHi - 1 . 7 . 0 [64] to determine those cross sections at $14 \mathrm{TeV}$ and $27 \mathrm{TeV}$. The renormalization and factorization scales are $\mu_{R}=m_{A}$ and $\mu_{F}=m_{A} / 4$, respectively, which have been shown to be the suitable choices. Scale uncertainties are determined by varying $\mu_{R}$ and $\mu_{F}$ such that $\mu_{R}, 4 \mu_{F} \in\left\{m_{A} / 2, m_{A}, 2 m_{A}\right\}$, with $1 / 2 \leq 4 \mu_{F} / \mu_{R}<2$. Although the $b$ quark is massless, the bottom Yukawa coupling is nonzero and renormalized in the $\overline{\mathrm{MS}}$ scheme. The LO cross section in the 5FS is proportional to $y_{b}^{2}$, while $\mathrm{N}^{k} \mathrm{LO}$ is proportional to $y_{b}^{2} \alpha_{S}^{k}$, with $y_{b} y_{t}$ terms vanishing order by order in perturbative QCD. In calculating the cross sections for both the 4FS and 5FS cases, we have used the PDF4LHC15_nlo_mC and PDF4LHC15_nnlo_ mc [65] PDFs, respectively. In order to combine both estimates of the cross section, we use the Santander matching criterion [66] such that

$$
\sigma^{\text {matched }}=\frac{\sigma^{4 \mathrm{FS}}+\alpha \sigma^{5 \mathrm{FS}}}{1+\alpha},
$$

where $\alpha=\ln \left(\frac{m_{A}}{m_{b}}\right)-2$. The matched cross section of the inclusive process lies between the 4FS and 5FS values, but closer to the 5FS value owing to the weight $\alpha$, which depends on the $C P$-odd Higgs mass. The uncertainties are combined as such: 
TABLE IV. The production cross sections, in $\mathrm{fb}$, of the $C P$-odd Higgs in the four-flavor scheme at NLO (in association with bottom quarks) and in the five-flavor scheme at NNLO along with the matched values at $\sqrt{s}=$ $14 \mathrm{TeV}$ and $\sqrt{s}=27 \mathrm{TeV}$ for benchmarks of Table I. The running $b$-quark mass, in $\mathrm{GeV}$, is also shown evaluated at the factorization and normalization scales, $\mu_{F}=\mu_{R}$ (in $\mathrm{GeV}$ ).

\begin{tabular}{|c|c|c|c|c|c|c|c|c|}
\hline \multirow[b]{2}{*}{ Model } & \multicolumn{2}{|c|}{$\sigma_{\mathrm{NLO}}^{4 \mathrm{FS}}(p p \rightarrow b \bar{b} A)$} & \multicolumn{2}{|c|}{$\sigma_{\mathrm{NNLO}}^{\mathrm{5FS}}(p p \rightarrow A)$} & \multicolumn{2}{|c|}{$\sigma^{\text {matched }}$} & $\underline{\mu_{F}}=\mu_{R}$ & $\bar{m}_{b}$ \\
\hline & $14 \mathrm{TeV}$ & $27 \mathrm{TeV}$ & $14 \mathrm{TeV}$ & $27 \mathrm{TeV}$ & $14 \mathrm{TeV}$ & $27 \mathrm{TeV}$ & \multicolumn{2}{|c|}{ (4FS only) } \\
\hline (a) & $649.4_{-5.8 \%}^{+4.1 \%}$ & $2388_{-5.4 \%}^{+2.1 \%}$ & $982.0_{-4.2 \%}^{+3.8 \%}$ & $3538_{-4.9 \%}^{+4.0 \%}$ & $881.0_{-4.7 \%}^{+3.9 \%}$ & $3188_{-5.1 \%}^{+3.5 \%}$ & 78.5 & 2.91 \\
\hline (b) & $996.9_{-5.8 \%}^{+4.3 \%}$ & $3926_{-5.0 \%}^{+1.9 \%}$ & $1565_{-3.6 \%}^{+3.5 \%}$ & $5963_{-4.1 \%}^{+3.6 \%}$ & $1400_{-4.3 \%}^{+3.7 \%}$ & $5369_{-4.4 \%}^{+3.1 \%}$ & 90.0 & 2.88 \\
\hline (c) & $521.0_{-6.3 \%}^{+4.5 \%}$ & $2201_{-4.6 \%}^{+1.9 \%}$ & $846.1_{-3.3 \%}^{+3.3 \%}$ & $3440_{-3.7 \%}^{+3.4 \%}$ & $755.4_{-4.2 \%}^{+3.6 \%}$ & $3094_{-3.2 \%}^{+3.7 \%}$ & 104.3 & 2.84 \\
\hline (d) & $497.0_{-7.0 \%}^{+5.2 \%}$ & $2200_{-4.7 \%}^{+2.9 \%}$ & $808.9_{-3.1 \%}^{+3.1 \%}$ & $3442_{-3.3 \%}^{+3.2 \%}$ & $724.2_{-4.1 \%}^{+3.7 \%}$ & $3105_{-3.7 \%}^{+3.1 \%}$ & 114.8 & 2.82 \\
\hline (e) & $165.2_{-7.0 \%}^{+5.4 \%}$ & $777.8_{-4.5 \%}^{+3.0 \%}$ & $277.2_{-2.9 \%}^{+3.0 \%}$ & $1247_{-3.1 \%}^{+3.0 \%}$ & $247.7_{-4.0 \%}^{+3.6 \%}$ & $1123_{-3.5 \%}^{+3.0 \%}$ & 128.1 & 2.79 \\
\hline (f) & $313.2_{-7.5 \%}^{+5.0 \%}$ & $1524_{-4.3 \%}^{+2.8 \%}$ & $530.8_{-2.8 \%}^{+2.9 \%}$ & $2493_{-3.0 \%}^{+2.9 \%}$ & $474.6_{-4.0 \%}^{+3.4 \%}$ & $2243_{-3.3 \%}^{+2.9 \%}$ & 138.9 & 2.77 \\
\hline (g) & $125.1_{-7.8 \%}^{+5.1 \%}$ & $649.6_{-4.7 \%}^{+3.4 \%}$ & $219.6_{-2.7 \%}^{+2.8 \%}$ & $1090_{-2.8 \%}^{+2.8 \%}$ & $195.9_{-4.0 \%}^{+3.4 \%}$ & $979.4_{-3.3 \%}^{+2.9 \%}$ & 153.1 & 2.75 \\
\hline (h) & $93.2_{-8.5 \%}^{+5.1 \%}$ & $555.0_{-4.9 \%}^{+3.5 \%}$ & $182.0_{-2.6 \%}^{+2.7 \%}$ & $944.2_{-2.7 \%}^{+2.7 \%}$ & $160.1_{-4.1 \%}^{+3.3 \%}$ & $848.0_{-3.3 \%}^{+2.90 \%}$ & 164.8 & 2.74 \\
\hline (i) & $92.8_{-8.4 \%}^{+5.2 \%}$ & $529.9_{-5.2 \%}^{+3.7 \%}$ & $164.1_{-2.6 \%}^{+2.6 \%}$ & $892.8_{-2.7 \%}^{+2.6 \%}$ & $146.8_{-4.0 \%}^{+3.3 \%}$ & $804.8_{-3.3 \%}^{+2.9 \%}$ & 177.8 & 2.72 \\
\hline (j) & $35.8_{-8.5 \%}^{+5.2 \%}$ & $213.5_{-5.4 \%}^{+3.8 \%}$ & $65.5_{-2.6 \%}^{+2.5 \%}$ & $371.9_{-2.6 \%}^{+2.6 \%}$ & $58.4_{-4.0 \%}^{+3.2 \%}$ & $334.1_{-3.3 \%}^{+2.9 \%}$ & 189.7 & 2.71 \\
\hline
\end{tabular}

$$
\delta \sigma^{\text {matched }}=\frac{\delta \sigma^{4 \mathrm{FS}}+\alpha \delta \sigma^{5 \mathrm{FS}}}{1+\alpha} .
$$

In Table IV, we give the NLO 4FS, NNLO 5FS, and matched cross sections at $14 \mathrm{TeV}$ and $27 \mathrm{TeV}$ for the ten benchmarks of Table I along with $\mu_{R}, \mu_{F}$ and the running $b$-quark mass in the 4FS case. Notice the dramatic increase in cross section in going from $14 \mathrm{TeV}$ to $27 \mathrm{TeV}$ due to the production of strongly interacting particles along with $A$. The cross sections have been checked with publicly available results [60] by a proper scaling of the bottom Yukawa coupling. In the MSSM, the tree-level Higgs Yukawa coupling to bottom quarks is given by

$$
y_{b b A}=\frac{\sqrt{2} m_{b}}{v} \tan \beta
$$

for $\tan \beta \gg 1$, where $v$ is the SM VEV. Besides QCD corrections, this Yukawa coupling receives SUSY-QCD corrections given, at one-loop level, by [67]

$$
\Delta_{b} \approx \frac{2 \alpha_{S}}{3 \pi} \frac{\mu m_{\tilde{g}}}{\max \left(m_{\tilde{g}}^{2}, m_{\tilde{b}_{1}}^{2}, m_{\tilde{b}_{2}}^{2}\right)} \tan \beta
$$

where $m_{\tilde{b}_{1,2}}$ and $m_{\tilde{g}}$ are the sbottom and the gluino masses. Taking this correction into consideration, one then needs to scale the SM $b \bar{b} h$ cross section by the square of

$$
y_{b b A} / y_{b b h} \sim \frac{\tan \beta}{1+\Delta_{b}}
$$

in order to obtain the MSSM cross section. However, $\Delta_{b}$ is negligible for our benchmarks due to heavy gluinos and sbottoms. In this case, the scaling only requires multiplying by $\tan ^{2} \beta$, with which we have found reasonable agreement with our results.

Due to its enhanced coupling to bottom quarks, the $C P$ odd Higgs preferentially decays to a $b \bar{b}$ pair, while the second-largest branching ratio is to a $\tau^{+} \tau^{-}$pair, as shown in Table V. In the MSSM, the branching ratio to $Z h$ is quite small and is not considered as a significant channel for discovery, at least at $14 \mathrm{TeV}$.

\section{VII. $C P$-ODD HIGGS SIGNATURE IN $\tau_{h} \tau_{h}$ FINAL STATE AT THE LHC}

We begin this section by a review of the experimental status of the MSSM $C P$-odd Higgs. The most recent constraints on the $C P$-odd Higgs mass come from Run 2 results from the ATLAS [68] and CMS [69] collaborations using $36 \mathrm{fb}^{-1}$ of data. ATLAS used low-scale benchmarks from Ref. [70] satisfying the light Higgs boson mass

TABLE V. The branching ratios of the $C P$-odd Higgs into standard-model particles for the benchmarks of Table I.

\begin{tabular}{lccc}
\hline \hline Model & $\mathrm{BR}(A \rightarrow b \bar{b})$ & $\mathrm{BR}\left(A \rightarrow \tau^{+} \tau^{-}\right)$ & $\mathrm{BR}(A \rightarrow Z h)$ \\
\hline (a) & 0.844 & 0.113 & 0.041 \\
(b) & 0.779 & 0.106 & 0.005 \\
(c) & 0.714 & 0.099 & 0.004 \\
(d) & 0.780 & 0.110 & 0.002 \\
(e) & 0.617 & 0.088 & 0.004 \\
(f) & 0.827 & 0.119 & 0.001 \\
(g) & 0.726 & 0.106 & 0.001 \\
(h) & 0.787 & 0.116 & 0.001 \\
(i) & 0.820 & 0.123 & $<0.001$ \\
(j) & 0.716 & 0.108 & 0.001 \\
\hline \hline
\end{tabular}


constraint and characterized by a small $\mu$ and SUSYbreaking scale (recommended by the LHC-HXSWG). Interpreted in the MSSM $\left(m_{\mathrm{h}}^{\bmod +}\right.$ model $),{ }^{1}$ the results exclude $\tan \beta>5$ for $m_{A}=250 \mathrm{GeV}$ and $\tan \beta>51$ for $m_{A}=1500 \mathrm{GeV}$. Due to the absence of light neutralinos in the spectrum, the $\mathrm{hMSSM}^{2}$ provides more stringent constraints because of a higher $A \rightarrow \tau \tau$ branching ratio. Thus, here $\tan \beta>1$ for $m_{A}=250 \mathrm{GeV}$ and $\tan \beta>42$ for $m_{A}=$ $1500 \mathrm{GeV}$ are excluded. CMS uses the same benchmarks, cross sections, and branching ratios of the $C P$-odd Higgs and arrives at similar exclusion limits, where $m_{A} \lesssim$ $250 \mathrm{GeV}$ is excluded for $\tan \beta>6$ and the exclusion contour reaches $1600 \mathrm{GeV}$ for $\tan \beta=60$. Projections for HL-LHC studies regarding the mass reach for the $C P$-odd Higgs in case no excess is found are available [74]. The benchmarks of Table I are not yet excluded by experiment and lie within the contour set for the HLLHC. It is worth stressing the fact that such interpretations of experimental results as mentioned above are carried out within models that are very different from the one we consider here. In addition, flavor-changing $B$-hadron decays constrain the MSSM parameter space, especially the charged Higgs and chargino masses, which have BSM contributions to such rare decays. The current limits on relevant decays are $\mathcal{B}(b \rightarrow s \gamma) \simeq(3.36 \pm 0.23) \times 10^{-4}$ [54], $\mathcal{B}\left(B_{s}^{0} \rightarrow \mu^{+} \mu^{-}\right)=\left(2.8_{-0.7}^{+0.8}\right) \times 10^{-9}, \quad$ and $\mathcal{B}\left(B^{0} \rightarrow\right.$ $\left.\mu^{+} \mu^{-}\right)<2.1 \times 10^{-10}$ [75]. For our benchmarks, $\mathcal{B}(b \rightarrow$ $s \gamma$ ) ranges from $3.22 \times 10^{-4}$ for point (i) to $3.37 \times 10^{-4}$ for point (c), while $\mathcal{B}\left(B_{s}^{0} \rightarrow \mu^{+} \mu^{-}\right)$ranges from $3.0 \times 10^{-9}$ for point (d) to $3.2 \times 10^{-9}$ for point (j), both of which are within the experimental error bars. As for $\mathcal{B}\left(B^{0} \rightarrow \mu^{+} \mu^{-}\right)$, values range from $9.8 \times 10^{-11}$ for point (d) to $1.0 \times 10^{-10}$ for point (j), which are below the experimental upper limit. Thus, all of our model points satisfy the current flavor constraints from experiment.
The signal we investigate consists of a $C P$-odd Higgs decaying to two hadronic taus and produced alongside two $b$ quarks, which can be tagged. Even in the 5FS, $b$-flavored jets can appear at the parton shower level, and so $b$ tagging is viable here too. In order to account for misidentified $b$-tagged jets, we require that our final states contain at least one $b$-tagged jet and two tau-tagged $\left(\tau_{h}\right)$ jets such that $p_{T}(b)>20 \mathrm{GeV},|\eta(b)|<2.5$, and $p_{T}\left(\tau_{h}\right)>15 \mathrm{GeV}$.

The standard-model backgrounds relevant to the final states considered here are $t \bar{t}, t+$ jets, $t+W / Z$, QCD multijets, dibosons, and $W, Z / \gamma^{*}+$ jets. The signal and SM backgrounds are simulated at LO with MadGraph52.6.3 interfaced with LHAPDF [76] and using the NNPDF30LO PDF set. The cross sections of the SM backgrounds are then normalized to their NLO values, while those of the signal are scaled to their matched values in Table IV. The parton-level events are passed to PYTHIA8 [77] for showering and hadronization. A fiveflavor MLM matching [78] is performed on the backgrounds to avoid double counting of jets at the shower level. Jets are clustered with FASTJET [79] using the anti$k_{t}$ algorithm [80] with jet radius 0.4. Detector simulation and event reconstruction are handled by DELPHES3.4.2 [81] using the new cards for the HL-LHC and HE-LHC generic detectors. The resulting files are read and analyzed with ROOT-6.16 [82].

Due to the smallness of the signal cross section in comparison to the SM backgrounds (especially following the selection criteria), we use boosted decision trees (BDTs) to separate the signal from the background. The type of BDT used here is known as an "adaptive BDT" (AdaBoost). Before giving a brief overview of BDTs, we list the kinematic variables used to help in discriminating the signal from the background:

(1) The total transverse mass of the ditau system is given by [83]

$$
m_{T}^{\text {tot }}=\sqrt{m_{T}^{2}\left(E_{T}^{\text {miss }}, \tau_{h 1}\right)+m_{T}^{2}\left(E_{T}^{\text {miss }}, \tau_{h 2}\right)+m_{T}^{2}\left(\tau_{h 1}, \tau_{h 2}\right)},
$$

where

$$
m_{T}(i, j)=\sqrt{2 p_{T}^{i} p_{T}^{j}\left(1-\cos \Delta \phi_{i j}\right)} .
$$

This variable has the best separating power, especially for heavier $C P$-odd Higgs mass.

(2) The hadronic ditau invariant mass, $m_{\tau_{h} \tau_{h}}$, has the same effect as $m_{T}^{\text {tot }}$ and in addition works well for low-mass signals.

\footnotetext{
${ }^{1}$ Here the top-squark mixing is fixed so that the lightest $C P$ even Higgs mass approximates the measured mass [71].

${ }^{2}$ Here the measured value of the Higgs boson $h$ is used to predict under certain assumptions the masses and couplings of the MSSM Higgs bosons [72,73].
}

(3) The angular separation $\Delta \phi\left(\tau_{h 1}, \tau_{h 2}\right)$ between the leading and subleading hadronic tau jets. For the signal, the variable is mostly peaked at $\Delta \phi\left(\tau_{h 1}, \tau_{h 2}\right)>2.8$, while it peaks for small values (near zero) for the background.

(4) The number of charged tracks associated with the leading tau, $N_{\text {tracks }}^{\tau}$. Due to its one- and three-prong decays, a tau can be identified by the tracks' charge multiplicities.

(5) Due to the presence of $b$-tagged jets, we use the number of such jets, $N_{\text {jet }}^{b}$, as a discriminating variable.

(6) Due to the rich jetty final states, we define the variable $\ln \left(p_{T}^{\text {jet }}\right)$ as 


$$
\ln \left(p_{T}^{\text {jet }}\right)=\left\{\begin{array}{ll}
\ln \left(p_{T}^{\text {jet }_{1}}\right) & \text { if } N_{\text {jets }} \geq 1 \\
0 & \text { if } N_{\text {jets }}=0
\end{array},\right.
$$

where $p_{T}^{\text {jet }}$ is the $p_{T}$ of the leading jet.

(7) The dijet transverse mass $m_{T}^{\text {dijet }}$ of the leading and subleading jets is a good discriminant against the QCD multijet, which tends to have a large value of this variable. If two jets cannot be found in an event, this variable is set to zero.

(8) The effective mass is defined as

$$
m_{\mathrm{eff}}=H_{T}+E_{T}^{\mathrm{miss}}+p_{T}\left(\tau_{h 1}\right)+p_{T}\left(\tau_{h 2}\right),
$$

where $H_{T}$ is the sum of the hadronic $p_{T}$ 's in an event, and $p_{T}\left(\tau_{h 1}\right)$ and $p_{T}\left(\tau_{h 2}\right)$ are the transverse momenta of the leading and subleading hadronic taus.

BDTs employ a multivariate analysis technique for a classification problem such as the one at hand. The aim here is to classify a certain set of events as belonging to the signal or the background by using a number of discriminating variables (1-8 listed above) to make the decision. The signal $(S)$ and background $(B)$ samples undergo two phases: the training and testing phases. In the first phase, the BDTs are trained on those samples using the available list of kinematic variables. The algorithm sorts those variables in descending order of separation power and chooses the variable that has the highest separation power to start the "root node." A cut is applied on this variable and events are split into left or right nodes depending on whether they were classified as signal or background. Afterwards, another variable (or sometimes the initiating variable) is chosen with a cut value which further splits down events into signal and background. The tree continues growing until a stopping criterion, such as the tree depth, is reached. The end layer of the tree contains the leaves, which host the events classified as signal (and given a value +1 ) or background (and given a value -1 ). During training, some signal events may be misclassified as background and vice versa. Those events will be given a weight factor and then enter in the second iteration of the training phase when the next tree starts forming. Those events are now given more attention thanks to the weight factor they carry. The training stops when the entire number of trees in the forest has been utilized. The number of trees and their depths are specified by the user in such a way as to maximize the separation between the signal and the background, where a larger depth generally produces a better separation.

The second phase is the testing phase, where the algorithm applies what it has learned on a statistically independent set of samples and outputs a new discriminating variable called the "BDT score" or "BDT response." An agreement between the performances of the training and testing phases is a sign of no overtraining occurring in the analysis. Such a situation can arise if one chooses too large of a tree depth while not having enough statistics in the samples. We have made sure that no overtraining of the samples has occurred throughout. The BDT implementation is carried out using ROOT's own TMVA (Toolkit for Multivariate Analysis) framework [84]. Depending on the samples, we set the number of trees to be in the range 120 to 200, the depth to 3, and the AdaBoost learning rate to 0.5. Many combinations of those parameters have been tried, and the one which gave the best result was considered. BDTs are very useful in classification problems where conventional linear cuts fail. To show that, we display in Fig. 3 distributions normalized to unity of four kinematic variables at $\sqrt{s}=27 \mathrm{TeV}$ for benchmark (a) of Table I. The purpose of such distributions is to help design event selection cuts which would allow better background rejection based on the shape of the distribution. One can clearly notice, for distributions in $\ln \left(p_{T}^{\text {jet }}\right), N_{\text {tracks }}^{\tau}$, and $\Delta \phi\left(\tau_{h 1}, \tau_{h 2}\right)$, that a conventional linear cut does not do the job, as it would lead to a poor signal-to-background ratio. This is where BDTs become powerful, since they employ nonlinear cuts in the multidimensional space of variables (thus the name "multivariate analysis"). On the other hand, a linear cut on $m_{T}^{\text {tot }}$ such that $m_{T}^{\text {tot }}>150 \mathrm{GeV}$ is reasonable but not sufficient to obtain a good signal-tobackground ratio. The BDT algorithm will run through distributions of such sort for the eight variables presented above and design unconventional cuts to obtain the best discrimination between the signal and the background.

After training and testing of the BDTs, we set a cut on the BDT score variable which would give us the minimum integrated luminosity for $\frac{S}{\sqrt{S+B}}$ at the $5 \sigma$ level of discovery. In general, this cut value is not common across all points, since each point is trained and tested separately along with the SM backgrounds, and so the distribution in BDT score differs from one point to another. We present in Fig. 4 the computed integrated luminosities, $\mathcal{L}$, as a function of the cut on the BDT score for both $14 \mathrm{TeV}$ (left panel) and $27 \mathrm{TeV}$ (right panel) machines. For $14 \mathrm{TeV}$, one can see that a drop in $\mathcal{L}$ occurs for BDT score $>0.3$, while at $27 \mathrm{TeV}$ the same is seen for BDT score $>0.2$.

Using the results from Fig. 4, we tabulate the lowest integrated luminosity for a $5 \sigma$ discovery at the HL-LHC and HE-LHC in Table VI for all our benchmarks. It is seen that half the benchmarks are discoverable at the HL-LHC. Thus, benchmark (d) is discoverable with an $\mathcal{L}$ as low as $866 \mathrm{fb}^{-1}$, while benchmark (f) requires an $\mathcal{L}$ close to the optimal integrated luminosity of $3000 \mathrm{fb}^{-1}$. However, all the benchmarks are discoverable at the HE-LHC, with some requiring an integrated luminosity smaller than $100 \mathrm{fb}^{-1}$, such as point (d), with $\mathcal{L}=50 \mathrm{fb}^{-1}$ for discovery. Point (j) requires the largest amount of data at $\sim 2600 \mathrm{fb}^{-1}$, which, however, is still much lower than the optimal integrated luminosity of $15 \mathrm{ab}^{-1}$ expected at the HE-LHC. 

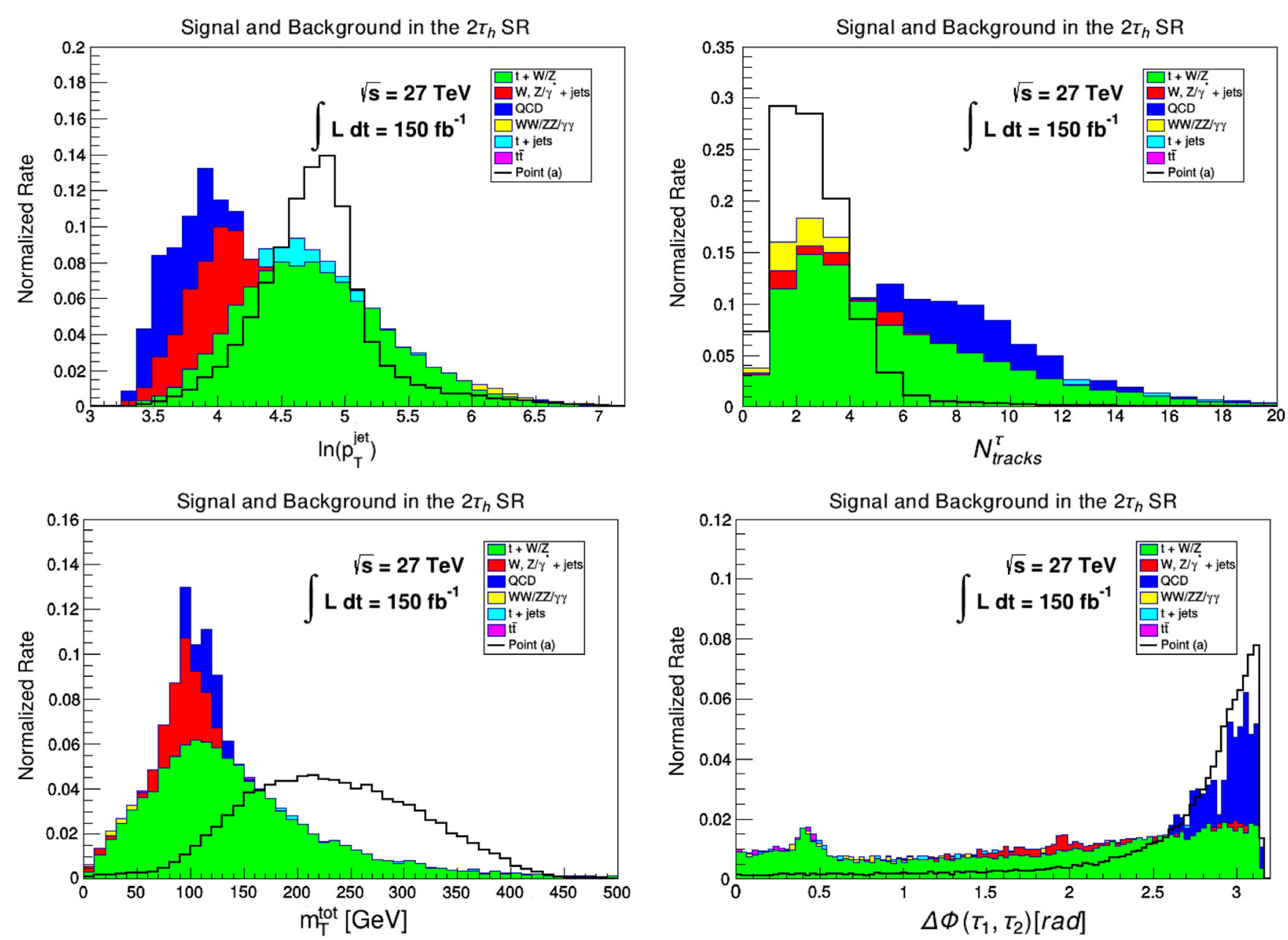

FIG. 3. Distributions normalized to the bin size of four kinematic variables for benchmark (a) at $27 \mathrm{TeV}: \ln \left(p_{T}^{\mathrm{jet}}\right)$ (top left), $N_{\text {tracks }}^{\tau}$ (top right), $m_{T}^{\text {tot }}$ (bottom left), and $\Delta \phi\left(\tau_{h 1}, \tau_{h 2}\right)$ (bottom right) in the $2 \tau_{h}$ signal region (SR).
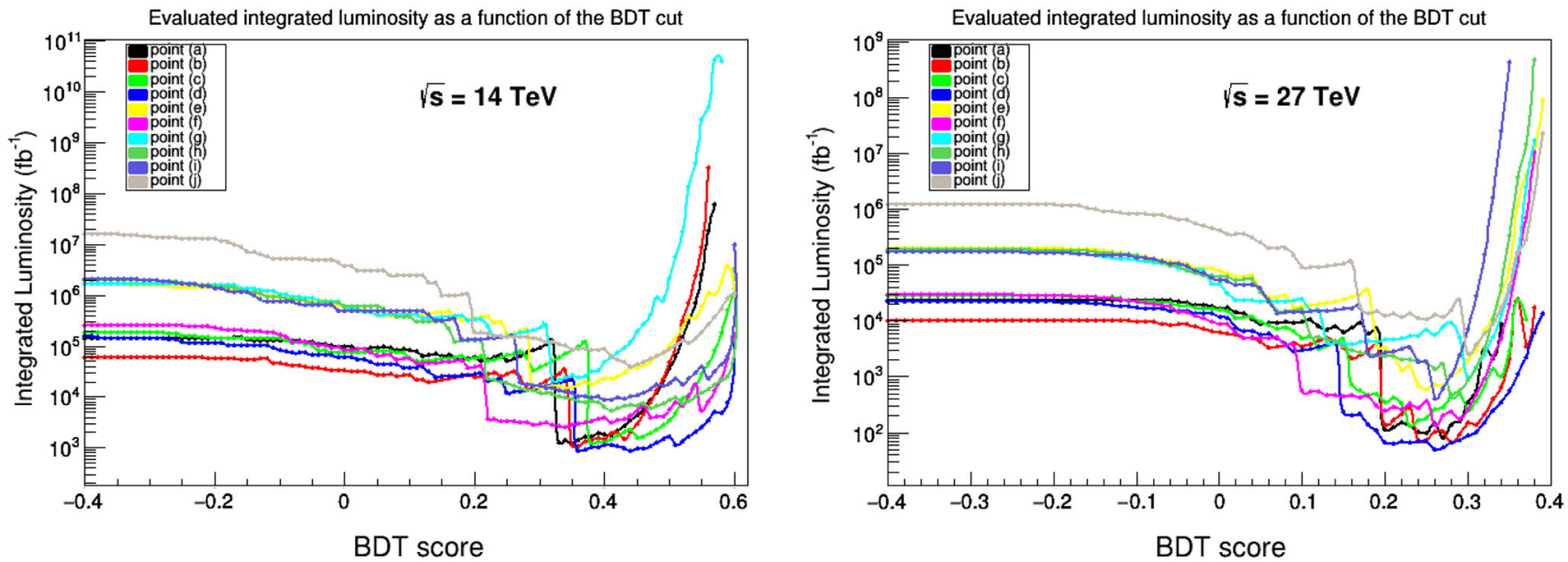

FIG. 4. The estimated integrated luminosities as a function of the BDT cut for the benchmarks of Table I at 14 TeV (left panel) and $27 \mathrm{TeV}$ (right panel). 
TABLE VI. Comparison between the estimated integrated luminosity $(\mathcal{L})$ in $\mathrm{fb}^{-1}$ for a $5 \sigma$ discovery at $14 \mathrm{TeV}$ (middle column) and $27 \mathrm{TeV}$ (right column) for the $C P$-odd Higgs following the selection criteria and BDT cut. An entry with an ellipsis means that the evaluated $\mathcal{L}$ is much greater than $3000 \mathrm{fb}^{-1}$.

\begin{tabular}{lcc}
\hline \hline & \multicolumn{2}{c}{$\mathcal{L}$ for $5 \sigma$ discovery in $2 \tau_{h}+b$-jets } \\
\cline { 2 - 3 } Model & $\mathcal{L}$ at $14 \mathrm{TeV}$ & $\mathcal{L}$ at $27 \mathrm{TeV}$ \\
\hline (a) & 1221 & 82 \\
(b) & 1102 & 67 \\
(c) & 1195 & 131 \\
(d) & 866 & 50 \\
(e) & $\ldots$ & 604 \\
(f) & 2598 & 136 \\
(g) & $\ldots$ & 952 \\
(h) & $\ldots$ & 420 \\
(i) & $\ldots$ & 412 \\
(j) & $\ldots$ & 2599 \\
\hline \hline
\end{tabular}

We show in Fig. 5 some distributions in the BDT score for points (a) and (d) at $14 \mathrm{TeV}$ and $27 \mathrm{TeV}$ for various representative integrated luminosities. For point (a), which is discoverable at both the HL-LHC and HE-LHC, we see that the signal is in excess over the background for $\mathcal{L}=150 \mathrm{fb}^{-1}$ at $27 \mathrm{TeV}$ (top-left panel), while a higher integrated luminosity is required for an excess at the HL-LHC_namely, $\quad \mathcal{L}=2000 \mathrm{fb}^{-1} \quad$ (top-right panel). Note that these integrated luminosities are not the minimum ones, with the latter presented in Table VI. The bottom two panels of Fig. 5 show the BDT scores for point (d) at $27 \mathrm{TeV}$ (left) and at $14 \mathrm{TeV}$ (right) for the same integrated luminosity of $200 \mathrm{fb}^{-1}$. Notice that the signal is in excess over the background at the HE-LHC contrary to the HLLHC case which is in accordance with the data of Table VI.

It is worth mentioning that in using BDTs, it is seen that better separation between signal and background occurs for points with higher $C P$-odd Higgs masses due to more energetic final states. However, a better outcome, i.e., a smaller integrated luminosity, is not always seen in those cases, since the price to be paid for larger masses is a falling cross section, which results in much higher integrated luminosity for discovery. Here we investigate the possibility if at higher masses the cross section, $\sigma \times \mathrm{BR}(A \rightarrow \tau \tau)$, can be maintained at a larger-than-usual value. This can be
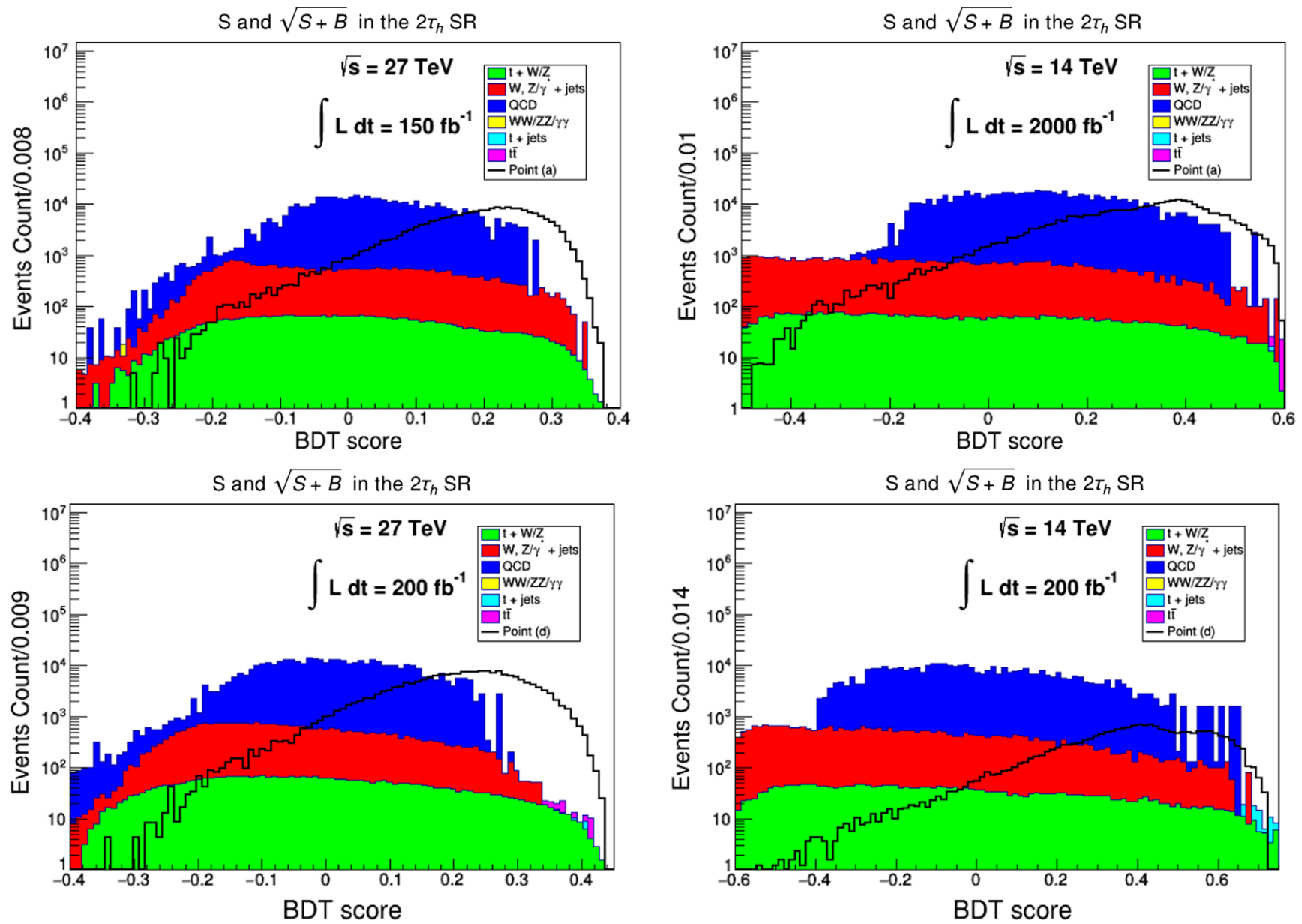

FIG. 5. Distributions in the BDT score for benchmarks (a) (top panels) and (d) (bottom panels) of Table I at 14 TeV (right panels) and $27 \mathrm{TeV}$ (left panels) in the $2 \tau_{h}$ signal region (SR) for various integrated luminosities. 

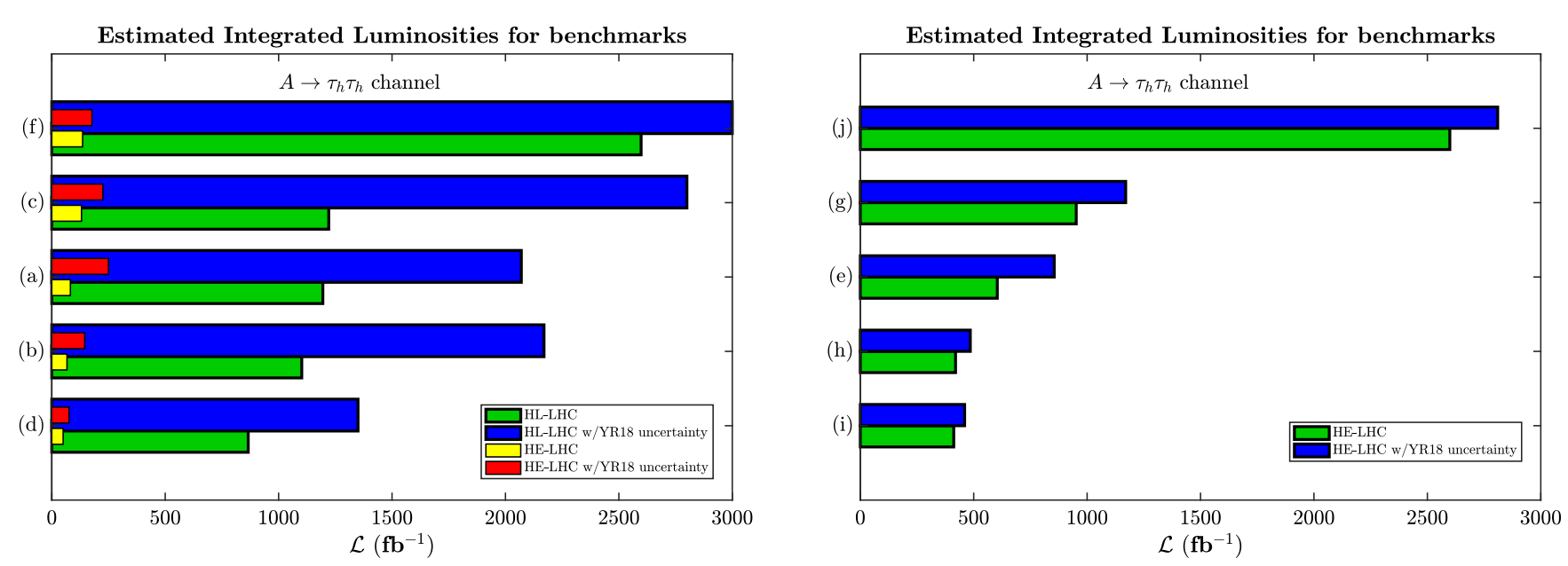

FIG. 6. Left panel: Five benchmarks of Table I that are discoverable at both the HL-LHC and the HE-LHC with and without the "YR18 uncertainties." Right panel: The remaining five benchmarks of Table I that are discoverable only at the HE-LHC with and without the "YR18 uncertainties."

achieved for a higher branching ratio and larger $\tan \beta$. Benchmark (b) has the largest cross section amongst all the points but requires the second-lowest integrated luminosity for discovery, with point (d) requiring the least. Now point (d) has a $C P$-odd Higgs mass $100 \mathrm{GeV}$ heavier than point (b), but it has a higher $\tan \beta$ and branching ratio, which makes up for the mass increase and keeps the cross section from falling too rapidly. Because of the effect of $\tan \beta$, the branching ratio, and more energetic final states, the separation between signal and background for point (d) is more pronounced, leading to the lowest integrated luminosity for discovery.

Given that the HE-LHC is expected to collect data at the rate of $820 \mathrm{fb}^{-1}$ per year [85] versus the rate at which the HL-LHC will collect data, the projected run-time for points (a)-(d) for discovery at the HL-LHC is $\sim 3$ to $\sim 4$ years, while point (f) requires $\sim 8$ years. The run-time is significantly decreased for the HE-LHC, where most of the points require $\sim 1$ to $\sim 6$ months, while point (d) $\sim 22$ days and point $(\mathrm{j}) \sim 3$ years.

Before concluding, we give an overview of the uncertainties one might expect and their impact on the estimated integrated luminosities at the HL-LHC and HE-LHC. One of the main challenges to overcome in experiment while analyzing data is presented by the systematic uncertainties. One would expect such uncertainties to decrease when the HL-LHC starts operation due to an increased dataset. It is also reasonable to assume that improvements on this front are expected by experimentalists working on the ATLAS and CMS detectors. Following experts' opinion in the HL-LHC and HE-LHC working groups at CERN [86,87], much of the systematic uncertainty is expected to drop by a factor of 2. These uncertainties are known as "YR18 systematic uncertainties." In the $A \rightarrow \tau_{h} \tau_{h}$ channel, systematic uncertainties due to the estimation of QCD jets to a $\tau_{h}$ fake background are dominant, especially in the low
$C P$-odd Higgs mass region. For higher masses, the leading uncertainty is from the reconstruction and identification of high transverse momentum $\tau_{h}$. In this analysis, we assume that the systematic uncertainties in the background and signal are $20 \%$ and $10 \%$, respectively. We give higher (lower) combined uncertainty for points with smaller (larger) Higgs mass. The integrated luminosity for a $5 \sigma$ discovery is reestimated after including the uncertainties using the signal significance [88]

$$
\begin{aligned}
\sigma= & {\left[2 \left((S+B) \ln \left[\frac{(S+B)\left(B+\Delta_{C}^{2}\right)}{B^{2}+(S+B) \Delta_{C}^{2}}\right]\right.\right.} \\
& \left.\left.-\frac{B^{2}}{\Delta_{C}^{2}} \ln \left[1+\frac{\Delta_{C}^{2} S}{B\left(B+\Delta_{C}^{2}\right)}\right]\right)\right]^{1 / 2},
\end{aligned}
$$

where $\Delta_{C}$ is the combined uncertainty in signal and background, $\Delta_{C}^{2}=\Delta_{S}^{2}+\Delta_{B}^{2}$. We show in Fig. 6 the estimated integrated luminosities before and after including the uncertainties for both the HL-LHC and HE-LHC. In the left panel, the five benchmarks discoverable at both machines are shown along with the "YR18 uncertainties," where at the HL-LHC, the integrated luminosities have increased by $\sim 1.5$ to $\sim 2.5$ times (in blue) compared to when no systematic uncertainties are present (in orange). At the HE-LHC, the increase is by $\sim 1.5$ to $\sim 4$ times (in red) compared to the case with no systematics (in yellow). The right panel shows the points that are discoverable only at the HE-LHC, along with the integrated luminosities before (in orange) and after (in blue) including uncertainties.

\section{CONCLUSIONS}

The large size of weak-scale supersymmetry implied by the Higgs boson mass at $\sim 125 \mathrm{GeV}$ has a direct implication for the discovery of supersymmetric dark matter. Thus, typically in high-scale SUGRA models with a large size of weak-scale supersymmetry, often the LSP neutralino is 
mostly a bino making an efficient annihilation of bino dark matter in the early Universe difficult and leading to an overabundance inconsistent with observation. Often coannihilation is utilized in this case, where a low-lying next-toLSP and the LSP together act to reduce the relic density within the observed limit. However, another branch of radiative breaking of the electroweak symmetry exists within the SUGRA model, where a large size of weakscale supersymmetry can coexist with a small Higgs mixing parameter $\mu$ (of the size of the electroweak scale). Such a situation can lead to a Higgsino-like LSP. As mentioned in the Introduction, models of this type are severely constrained by simultaneous satisfaction of dark matter relic density and by the spin-independent proton-DM scattering cross section limits in direct-detection experiments. However, such models can be made viable if the dark matter is multicomponent. Thus, in this work we considered an extension of the MSSM/SUGRA gauge group with a $U(1)_{X}$ of the hidden sector, where the $U(1)_{X}$ and $U(1)_{Y}$ have kinetic and Stueckelberg mass mixings. Further, we assume that the hidden sector is populated with chiral matter leading to a Dirac fermion, which acts as the second component of dark matter and makes up the dark matter deficit in the relic density. One implication of the model is the existence of a relatively light $C P$-odd Higgs boson $A$, as well as relatively light $H$ and $H^{ \pm}$, which have masses of the electroweak size. We investigate a set of benchmarks for the extended model and show that the $\mathrm{CP}$-odd Higgs boson in models of this type is observable in the next-generation collider experiments. Specifically, we investigate the discovery potential of a $C P$-odd Higgs in the $\tau_{h} \tau_{h}$ final state at the HL-LHC and HE-LHC. It is seen that a $C P$-odd Higgs with a mass up to $450 \mathrm{GeV}$ for $\tan \beta \leq 12$ may be discoverable at the HL-LHC. The discovery reaches $750 \mathrm{GeV}$ at the HE-LHC with an integrated luminosity of $\sim 2600 \mathrm{fb}^{-1}$, which is just a fraction of the optimal luminosity of $15 \mathrm{ab}^{-1}$ that the HE-LHC can deliver. With the optimal luminosity, the mass reach of the HE-LHC for the $C P$-odd Higgs mass will certainly extend far above $750 \mathrm{GeV}$. It is also shown that a significant part of the parameter space of the extended model can be probed in the next-generation direct-detection experiments such as XENONnT and LUX-ZEPLIN.

The analysis we carry out in this work is novel on several fronts. Thus, a main problem of any supersymmetric model with a light Higgs is that the LSP will be mostly a Higgsino which annihilates copiously in the early Universe, and the relic density of the surviving LSP falls below the desired relic density of cold dark matter. Thus, a theory model with light Higgs cannot produce the desired amount of cold dark matter and is incomplete. This deficiency is removed in the model presented. Thus, from the point of view of a complete model, naturalness in the form of a small $\mu$ with a neutralino, mostly a Higgsino, calls for a two-component (and, more generally, multicomponent) dark matter and predicts relatively light Higgses. Thus, even though the extra component of dark matter has interactions too weak to be produced at the LHC, the model can be tested through the discovery of light Higgses. In this work, we have also discussed the discovery potential of a $C P$-odd Higgs at the HL-LHC and HE-LHC, where studies for the latter have not been carried out so far in the literature. In the analysis, we have used BDTs for improved signal-to-background significance. In brief, even though the hidden-sector DM candidate does not produce a direct signature at colliders, the possible discovery of a light $C P$-odd Higgs would point towards a multicomponent DM scenario for the reason discussed above.

\section{ACKNOWLEDGMENTS}

The analysis presented here was done using the resources of the high-performance Cluster353 at the Advanced Scientific Computing Initiative (ASCI) and the Discovery Cluster at Northeastern University. This research was supported in part by NSF Grant No. PHY-1620575.
[1] G. Aad et al. (ATLAS Collaboration), Phys. Lett. B 716, 1 (2012).

[2] S. Chatrchyan et al. (CMS Collaboration), Phys. Lett. B 716, 30 (2012).

[3] B. W. Lee, C. Quigg, and H. B. Thacker, Phys. Rev. Lett. 38, 883 (1977).

[4] P. Nath, Supersymmetry, Supergravity, and Unification (Cambridge University Press, Cambridge, England, 2016).

[5] S. Akula, B. Altunkaynak, D. Feldman, P. Nath, and G. Peim, Phys. Rev. D 85, 075001 (2012).
[6] A. Arbey, M. Battaglia, A. Djouadi, and F. Mahmoudi, J. High Energy Phys. 09 (2012) 107.

[7] S. Akula, P. Nath, and G. Peim, Phys. Lett. B 717, 188 (2012).

[8] G. Degrassi, S. Di Vita, J. Elias-Miro, J. R. Espinosa, G. F. Giudice, G. Isidori, and A. Strumia, J. High Energy Phys. 08 (2012) 098.

[9] A. V. Bednyakov, B. A. Kniehl, A. F. Pikelner, and O. L. Veretin, Phys. Rev. Lett. 115, 201802 (2015).

[10] N. Aghanim et al. (Planck Collaboration), arXiv:1807 .06209 . 
[11] K. L. Chan, U. Chattopadhyay, and P. Nath, Phys. Rev. D 58, 096004 (1998).

[12] U. Chattopadhyay, A. Corsetti, and P. Nath, Phys. Rev. D 68, 035005 (2003).

[13] S. Akula, M. Liu, P. Nath, and G. Peim, Phys. Lett. B 709, 192 (2012).

[14] J. L. Feng, K. T. Matchev, and T. Moroi, Phys. Rev. Lett. 84, 2322 (2000).

[15] H. Baer, C. Balazs, A. Belyaev, T. Krupovnickas, and X. Tata, J. High Energy Phys. 06 (2003) 054.

[16] D. Feldman, G. Kane, E. Kuflik, and R. Lu, Phys. Lett. B 704, 56 (2011).

[17] G. G. Ross, K. Schmidt-Hoberg, and F. Staub, J. High Energy Phys. 03 (2017) 021.

[18] D. Feldman, Z. Liu, P. Nath, and G. Peim, Phys. Rev. D 81, 095017 (2010).

[19] B. Holdom, Phys. Lett. 166B, 196 (1986).

[20] B. Holdom, Phys. Lett. B 259, 329 (1991).

[21] B. Kors and P. Nath, J. High Energy Phys. 07 (2005) 069; 12 (2004) 005; Phys. Lett. B 586, 366 (2004).

[22] K. Cheung and T. C. Yuan, J. High Energy Phys. 03 (2007) 120; D. Feldman, Z. Liu, and P. Nath, J. High Energy Phys. 11 (2006) 007; D. Feldman, P. Fileviez Perez, and P. Nath, J. High Energy Phys. 01 (2012) 038; W. Z. Feng, P. Nath, and G. Peim, Phys. Rev. D 85, 115016 (2012).

[23] D. Feldman, Z. Liu, and P. Nath, Phys. Rev. D 75, 115001 (2007).

[24] W. C. Huang, H. Ishida, C. T. Lu, Y. L. S. Tsai, and T. C. Yuan, Eur. Phys. J. C 78, 613 (2018).

[25] C. T. Huang, R. Ramos, V. Q. Tran, Y. L. S. Tsai, and T. C. Yuan, arXiv:1905.02396.

[26] N. Arkani-Hamed, T. Han, M. Mangano, and L. T. Wang, Phys. Rep. 652, 1 (2016).

[27] M. Mangano, CERN Yellow Rep. Monogr. 3, 1 (2017).

[28] M. Benedikt and F. Zimmermann, Nucl. Instrum. Methods Phys. Res., Sect. A 907, 200 (2018).

[29] F. Zimmermann, Nucl. Instrum. Methods Phys. Res., Sect. A 909, 33 (2018).

[30] J. Kalinowski, S. F. King, and J. P. Roberts, J. High Energy Phys. 01 (2009) 066; L. Basso, B. O'Leary, W. Porod, and F. Staub, J. High Energy Phys. 09 (2012) 054; G. Bélanger, J. Da Silva, U. Laa, and A. Pukhov, J. High Energy Phys. 09 (2015) 151; P. Athron, A. W. Thomas, S. J. Underwood, and M. J. White, Phys. Rev. D 95, 035023 (2017).

[31] A. Aboubrahim and P. Nath, Phys. Rev. D 99, 055037 (2019).

[32] M. Klasen, F. Lyonnet, and F. S. Queiroz, Eur. Phys. J. C 77, 348 (2017).

[33] K. J. Bae, H. Baer, A. Lessa, and H. Serce, J. Cosmol. Astropart. Phys. 10 (2014) 082.

[34] D. J. E. Marsh, Phys. Rep. 643, 1 (2016).

[35] L. Hui, J. P. Ostriker, S. Tremaine, and E. Witten, Phys. Rev. D 95, 043541 (2017).

[36] J. Halverson, C. Long, and P. Nath, Phys. Rev. D 96, 056025 (2017).

[37] A. Aboubrahim and P. Nath, Phys. Rev. D 98, 095024 (2018).

[38] A. Aboubrahim and P. Nath, Phys. Rev. D 98, 015009 (2018).
[39] A. Aboubrahim and P. Nath, Phys. Rev. D 96, 075015 (2017).

[40] A. Aboubrahim, P. Nath, and A. B. Spisak, Phys. Rev. D 95, 115030 (2017).

[41] T. Han, A. Ismail, and B. Shams Es Haghi, Phys. Lett. B 793, 354 (2019).

[42] H. Baer, V. Barger, J. S. Gainer, D. Sengupta, H. Serce, and X. Tata, Phys. Rev. D 98, 075010 (2018).

[43] F. Staub, Comput. Phys. Commun. 185, 1773 (2014).

[44] F. Staub, Adv. High Energy Phys. 2015, 840780 (2015).

[45] W. Porod, Comput. Phys. Commun. 153, 275 (2003).

[46] W. Porod and F. Staub, Comput. Phys. Commun. 183, 2458 (2012).

[47] A. Pukhov, arXiv:hep-ph/0412191.

[48] E. E. Boos, M. N. Dubinin, V. A. Ilyin, A. E. Pukhov, and V. I. Savrin, arXiv:hep-ph/9503280.

[49] G. Bélanger, F. Boudjema, A. Pukhov, and A. Semenov, Comput. Phys. Commun. 192, 322 (2015).

[50] J. Alwall, R. Frederix, S. Frixione, V. Hirschi, F. Maltoni, O. Mattelaer, H.-S. Shao, T. Stelzer, P. Torrielli, and M. Zaro, J. High Energy Phys. 07 (2014) 079.

[51] A. H. Chamseddine, R. Arnowitt, and P. Nath, Phys. Rev. Lett. 49, 970 (1982); P. Nath, R. L. Arnowitt, and A. H. Chamseddine, Nucl. Phys. B227, 121 (1983); L. J. Hall, J. D. Lykken, and S. Weinberg, Phys. Rev. D 27, 2359 (1983).

[52] S. Akula and P. Nath, Phys. Rev. D 87, 115022 (2013).

[53] H. Bahl, S. Liebler, and T. Stefaniak, Eur. Phys. J. C 79, 279 (2019).

[54] M. Tanabashi et al. (Particle Data Group), Phys. Rev. D 98, 030001 (2018).

[55] G. Aad et al. (ATLAS Collaboration), arXiv:1903.06248.

[56] E. Aprile et al. (XENON Collaboration), Phys. Rev. Lett. 121, 111302 (2018).

[57] D. S. Akerib et al. (LUX-ZEPLIN Collaboration), arXiv:1802.06039.

[58] D. S. Akerib et al. (LUX Collaboration), Phys. Rev. Lett. 118, 021303 (2017).

[59] X. Cui et al. (PandaX-II Collaboration), Phys. Rev. Lett. 119, 181302 (2017).

[60] D. de Florian et al. (LHC Higgs Cross Section Working Group), arXiv:1610.07922.

[61] A. Alloul, N. D. Christensen, C. Degrande, C. Duhr, and B. Fuks, Comput. Phys. Commun. 185, 2250 (2014).

[62] C. Degrande, C. Duhr, B. Fuks, D. Grellscheid, O. Mattelaer, and T. Reiter, Comput. Phys. Commun. 183, 1201 (2012).

[63] C. Degrande, Comput. Phys. Commun. 197, 239 (2015).

[64] R. V. Harlander, S. Liebler, and H. Mantler, Comput. Phys. Commun. 184, 1605 (2013).

[65] J. Butterworth et al., J. Phys. G 43, 023001 (2016).

[66] R. Harlander, M. Kramer, and M. Schumacher, arXiv:1112 .3478 .

[67] J. Baglio and A. Djouadi, J. High Energy Phys. 03 (2011) 055 .

[68] M. Aaboud et al. (ATLAS Collaboration), J. High Energy Phys. 01 (2018) 055.

[69] A. M. Sirunyan et al. (CMS Collaboration), J. High Energy Phys. 09 (2018) 007.

[70] S. Heinemeyer et al. (LHC Higgs Cross Section Working Group), arXiv:1307.1347. 
[71] M. Carena, S. Heinemeyer, O. Stå 1, C. E. M. Wagner, and G. Weiglein, Eur. Phys. J. C 73, 2552 (2013).

[72] A. Djouadi, L. Maiani, G. Moreau, A. Polosa, J. Quevillon, and V. Riquer, Eur. Phys. J. C 73, 2650 (2013).

[73] E. Bagnaschi et al., Report No. LHCHXSWG-2015-002.

[74] ATLAS and CMS Collaborations, arXiv:1902.10229.

[75] M. Aaboud et al. (ATLAS Collaboration), J. High Energy Phys. 04 (2019) 098.

[76] A. Buckley, J. Ferrando, S. Lloyd, K. Nordström, B. Page, M. Rüfenacht, M. Schönherr, and G. Watt, Eur. Phys. J. C 75, 132 (2015).

[77] T. Sjöstrand, S. Ask, J. R. Christiansen, R. Corke, N. Desai, P. Ilten, S. Mrenna, S. Prestel, C. O. Rasmussen, and P. Z. Skands, Comput. Phys. Commun. 191, 159 (2015).

[78] M. L. Mangano, M. Moretti, F. Piccinini, and M. Treccani, J. High Energy Phys. 01 (2007) 013.

[79] M. Cacciari, G. P. Salam, and G. Soyez, Eur. Phys. J. C 72, 1896 (2012).
[80] M. Cacciari, G. P. Salam, and G. Soyez, J. High Energy Phys. 04 (2008) 063.

[81] J. de Favereau, C. Delaere, P. Demin, A. Giammanco, V. Lemaître, A. Mertens, and M. Selvaggi (DELPHES 3 Collaboration), J. High Energy Phys. 02 (2014) 057.

[82] I. Antcheva et al., Comput. Phys. Commun. 182, 1384 (2011).

[83] The ATLAS Collaboration, Report No. ATLAS-CONF2016-085.

[84] P. Speckmayer, A. Hocker, J. Stelzer, and H. Voss, J. Phys. Conf. Ser. 219, 032057 (2010).

[85] M. Benedikt, F. Zimmermann et al. (FCC Collaboration), at the HL-LHC/HE-LHC Physics Workshop, (CERN, Geneva, Switzerland, 2017).

[86] M. Cepeda et al. (Physics of the HL-LHC Working Group), arXiv:1902.00134.

[87] X. Cid Vidal et al. (Working Group 3), arXiv:1812.07831

[88] G. Cowan, K. Cranmer, E. Gross, and O. Vitells, Eur. Phys. J. C 71, 1554 (2011); 73, 2501 (2013). 